Revue des patrimoines

$17 \mid 2011$

Les patrimoines de l'enseignement supérieur

\title{
La constitution de l'espace universitaire parisien (XIIIe - XVIIIe siècle) : jalons pour la redécouverte d'un patrimoine
}

Retour sur un patrimoine parisien méconnu : les espaces de transmission du savoir (III)

\section{Christian Hottin}

\section{(2) OpenEdition}

\section{Journals}

Édition électronique

URL : http://journals.openedition.org/insitu/11310

DOI : $10.4000 /$ insitu. 11310

ISSN : 1630-7305

Éditeur

Ministère de la culture

Référence électronique

Christian Hottin, « La constitution de l'espace universitaire parisien (XIIle - XVIIIe siècle) : jalons pour la redécouverte d'un patrimoine $»$, In Situ [En ligne], 17 | 2011, mis en ligne le 12 septembre 2014, consulté le 19 avril 2019. URL : http://journals.openedition.org/insitu/11310 ; DOI : 10.4000/ insitu. 11310

Ce document a été généré automatiquement le 19 avril 2019

\section{(i) $8=$

In Situ Revues des patrimoines est mis à disposition selon les termes de la licence Creative Commons Attribution - Pas d'Utilisation Commerciale - Pas de Modification 4.0 International. 


\section{La constitution de l'espace universitaire parisien (XIIIe - XVIIIe siècle) : jalons pour la redécouverte d'un patrimoine}

Retour sur un patrimoine parisien méconnu : les espaces de transmission du savoir (III)*

\section{Christian Hottin}

« Paris, mère des sciences, comme une autre Cariath Sepher ${ }^{1}$, cité des lettres, brille d'un éclat précieux. Grande sans doute, elle fait attendre d'elle de plus grandes choses, grâce à ceux qui apprennent et à ceux qui enseignent »

Bulle Parens scientiarum universitas (1231)

1 Le système de l'enseignement supérieur français, tel qu'il a été institué pendant la Révolution et sous l'Empire, localisé essentiellement à Paris, avec ses grandes écoles, ses facultés et les bâtiments les abritant est beaucoup moins qu'on pourrait le penser une création ex nihilo. Il emprunte son territoire et nombre de ses édifices à l'ancienne Université de Paris et aux divers établissements nés hors de son sein mais ayant grandi dans son orbite au cours de l'époque moderne. S'interroger dans un premier temps sur la genèse de cet espace universitaire, dont les racines remontent au Moyen Âge, n'est pas seulement l'occasion de mettre en perspective, dans la longue durée de l'institution, les stratégies de localisation développées par le pouvoir politique ou par les divers établissements aux XIX ${ }^{\mathrm{e}}$ et $\mathrm{XX}^{\mathrm{e}}$ siècles. En effet, analyser la formation de ce territoire, de ce quartier des écoles, ou encore de ce quartier Latin, donne des clefs pour la compréhension de certains comportements, de certaines représentations de l'espace, qui, puissamment enracinés dans les mentalités collectives des membres des communautés, vont peser sur les choix de localisation tout au long de la période contemporaine. L'attachement manifesté à tous les niveaux de décision pour cette portion de la rive 
gauche de la Seine trouve, pour partie au moins, son explication dans les liens tissés aux temps plus anciens entre l'institution enseignante et ce territoire. Un temps rompus dans les premières années de la Révolution, ces liens se sont rapidement renoués, avec l'installation de nombreuses structures nouvelles d'enseignement dans des locaux de l'ancien monde universitaire.

2 La persistance de l'ancienne Université de Paris sur les nouvelles et multiples universités parisiennes n'est pas uniquement une ombre portée immatérielle, un esprit de quartier attaché à la rive gauche par une longue histoire : c'est également un ensemble de traces matérielles bien tangibles, parfois difficiles à discerner dans le tissu urbain actuel, soit qu'elles soient insérées dans de nouvelles constructions universitaires, soit qu'elles aient été, parfois de très longue date, renvoyées au statut de propriétés privées. Considéré dans la longue durée de cinq siècles de créations et de transformations architecturales, le patrimoine immobilier constitué par l'Ancienne Université de Paris apparaît de fait fort considérable. Une grande part de celui-ci a été détruite au XIX siècle, lors des travaux de modernisation et d'assainissement de la rive gauche. On s'est déjà attaché à montrer, dans de précédentes livraisons d'In situ, que des témoignages importants subsistaient toutefois, souvent liés à des établissements extérieurs à l'Université et ayant partie liée avec l'élaboration de méthodes nouvelles d'enseignement à la fin de l'époque moderne. À travers une vue cavalière de l'histoire spatiale de l'Université, on s'attachera à mettre ici en évidence l'importance de ces traces - quand bien même les édifices restants auraient perdu tout lien avec l'institution universitaire. Un patrimoine caché, mais d'une remarquable densité, en somme... Enfin, il conviendra de s'intéresser de plus près à la spécificité de cet espace universitaire parisien, en esquissant des comparaisons avec d'autres métropoles savantes européennes.

\section{La formation de l'espace universitaire parisien au Moyen Âge : genèse et enracinement}

3 L'idée d'une université presque totalement immatérielle, formée de la seule universitas ( i.e. communauté) des maîtres et étudiants s'impose à tous pour décrire la situation de cette institution à l'époque de sa fondation et de son précoce apogée, soit au XIII ${ }^{e}$ siècle. Stephen d'Irsay a synthétisé en une phrase le paradoxe de cette apparente faiblesse qui constitue en fait la force principale de l'université :

«La vraie université médiévale, dans sa belle époque du XIII ${ }^{\mathrm{e}}$ siècle, n'a pas de bâtiments spéciaux, pas de fonctionnaires salariés. Cette pauvreté apparente fut sa force; elle n'étalait pas sa puissance dans des palais, dans des gloires matérielles, mais elle n'était pas moins redoutable par sa cohésion, son esprit de corps, par ce manque de stabilité qui lui permettait de se déplacer à son gré, bâton en main, tout comme un pauvre pèlerin sans biens terrestres $»^{2}$.

4 Cette insistance portée sur la communauté au détriment de l'architecture se retrouve par exemple sous la plume de Simone Roux, qui compare l'université aux autres corps de métiers ${ }^{3}$, ayant comme eux ses privilèges propres, et devant pour travailler occuper certains lieux, mais sans pour autant se définir par ces bâtiments : «le corps social [au Moyen Âge] n'imposait pas sa présence matérielle par la définition d'un lieu précis et délimité qui représenterait totalement et uniquement l'institution $»^{4}$.

Ce disant, elle affirme toutefois que la rive gauche est bien définie comme « l'Université », le « monde des écoles », par opposition à la Cité ou à la Ville, comme l'explicite la légende 
du plan de Truschet et Hoyau: «Icy et le vray pourtraict naturel de la Ville, Cité, Université (...) $»^{5}$. De fait, l'identification de la rive gauche de la Seine à l'espace universitaire, l'idée même d'un "quartier Latin» sont des notions tout autant constitutives et autrement plus durables d'une définition de l'institution universitaire que son caractère immatériel.

Décrite comme sans bâtiments spécifiques, l'Université naissante n'en est pas moins enracinée dans l'espace, un espace essentiellement urbain, qui s'oppose à celui des écoles monastiques. Dès le XII ${ }^{e}$ siècle, Abélard fait état de cette conjonction entre activité intellectuelle et vie urbaine: alors qu'ils sont au Paraclet ${ }^{6}$, écrit-il, ses étudiants s'ennuient et regrettent la ville. Cette prise de conscience d'un lien entre étude et ville est un des aspects du développement de la société urbaine en même temps qu'une des modalités de définition du travail intellectuel, qui se doit d'être exercé en ville, par opposition au travail manuel, qui peut être indifféremment citadin ou rustique ${ }^{7}$.

7 Absence de locaux particuliers, mais aussi identification au monde urbain et à une portion spécifique de l'espace parisien, ces trois caractères sont propres au monde des écoles du XII ${ }^{e}$ siècle, trois facteurs essentiels à la compréhension de la genèse de l'espace universitaire parisien, prélude à son enracinement sur les pentes de la Montagne SainteGeneviève.

\section{Le XII ${ }^{\mathrm{e}}$ siècle : les prémisses d'un espace propre}

Dès les premières années du siècle, les écoles du cloitre de la Cité, l'abbaye de Saint-Victor et celle de Sainte-Geneviève, apparaissent comme les principaux foyers d'éducation : ces trois implantations délimitent pour longtemps le territoire de l'institution enseignante. Si l'Abbaye Saint-Victor s'impose alors comme un centre intellectuel important, grâce à Guillaume de Champeaux ${ }^{8}$, elle n'en reste pas moins étroitement soumise à l'autorité épiscopale. Ce n'est pas le cas de Sainte-Geneviève (fig. 1), abbaye nullius, c'est-à-dire qui ne dépend de personne. Elle échappe au pouvoir de l'évêque et ne relève que du pape pour les questions ecclésiastiques. En outre, son domaine s'étend loin sur le versant nord de la Montagne Sainte-Geneviève, presque jusqu'aux berges de la Seine. 


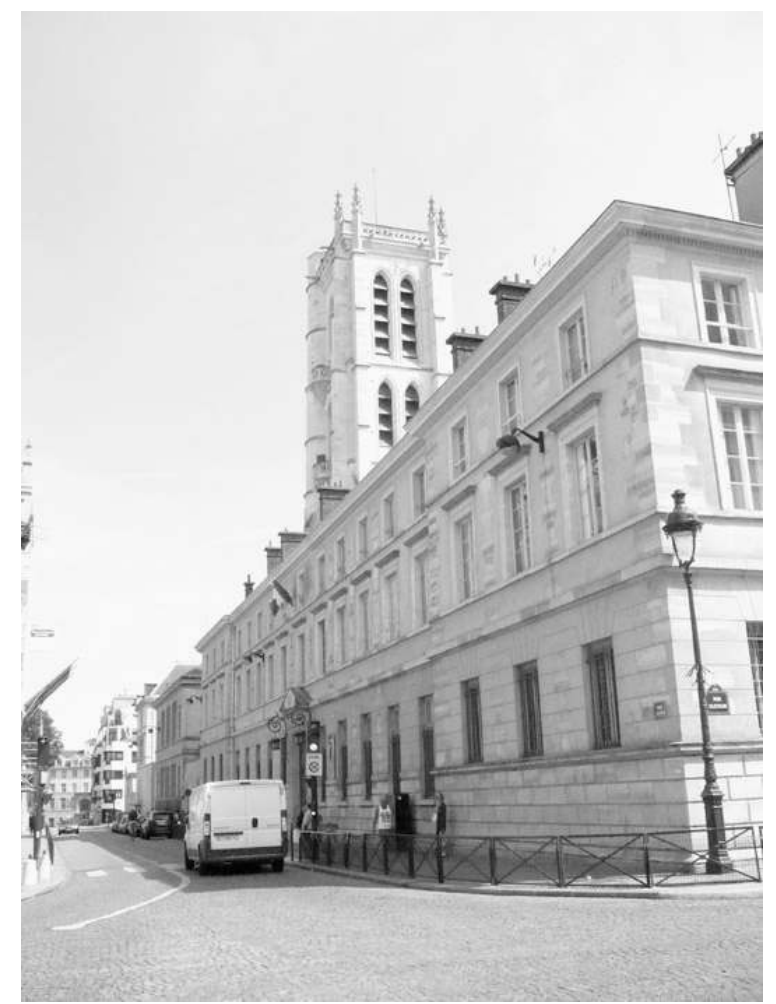

L'Abbaye Sainte-Geneviève, actuel Lycée Henri IV. Au fond, la tour de Clovis. PHOT. HOTTIN, CHRISTIAN, 2014. (C) HOTTIN, CHRISTIAN.

Cette situation juridique et géographique particulière est à l'origine du mouvement de translation qui conduit, au début du XII siècle, maîtres et étudiants à s'établir d'abord sur le Petit Pont et dans les rues avoisinantes, puis sur les pentes de la colline. Le plus célèbre d'entre eux est Abélard, qui trouve ainsi un moyen d'échapper aux censures de l'archidiacre de Notre-Dame et de délivrer plus librement son enseignement ${ }^{10}$. Ce sont bientôt plus de vingt maitres qui exercent leur magistère dans un espace compris entre le Petit Pont et la place Maubert. Le quartier acquiert alors cette ambiance particulière de jeunesse et de dynamisme intellectuel qu'évoque Jean de Salisbury, fasciné par la foule d'étudiants qui peuple les lieux ${ }^{11}$. Dans la deuxième moitié du siècle, alors que les écoles de Paris connaissent un moindre rayonnement, cette géographie demeure: le terrain propice aux études se situe entre la cathédrale et les deux abbayes, des rives du fleuve aux pentes de la colline.

\section{La cristallisation des années 1250-1350 : artiens, mendiants et collèges}

Tandis que se mettent en place les fondements institutionnels de l'université (privilège du For en 1200, statuts de Robert de Courson en 1215, bulles Super Speculam et Parens Scientiarum en 1219 et 1231), la rive gauche devient définitivement le lieu de vie et d'étude des artiens, qui quittent la Cité en 1219 à la faveur d'un conflit avec l'évêque. Ils disposent en effet sur les terres de Sainte-Geneviève d'un refuge qui leur appartient depuis le début de siècle : en 1202, Mathieu de Montmorency et sa femme Mathilde de Garlande leur ont 
cédé le Clos Mauvoisin, qu'ils tiennent des génovéfains et qui est situé près de SaintJulien-le-Pauvre. Les artiens s'établissent dans les lieux, et plus particulièrement dans la rue du Fouarre (fig. 2), située non loin de l'emplacement de l'actuelle rue Galande ${ }^{12}$. Mais le territoire de l'Université va gagner en superficie et en densité d'occupation principalement grâce à l'installation sur la rive gauche de deux types d'établissements nécessairement pourvus en locaux, alors même que l'université n'en possède quasiment pas : les ordres mendiants et les collèges.

Figure 2

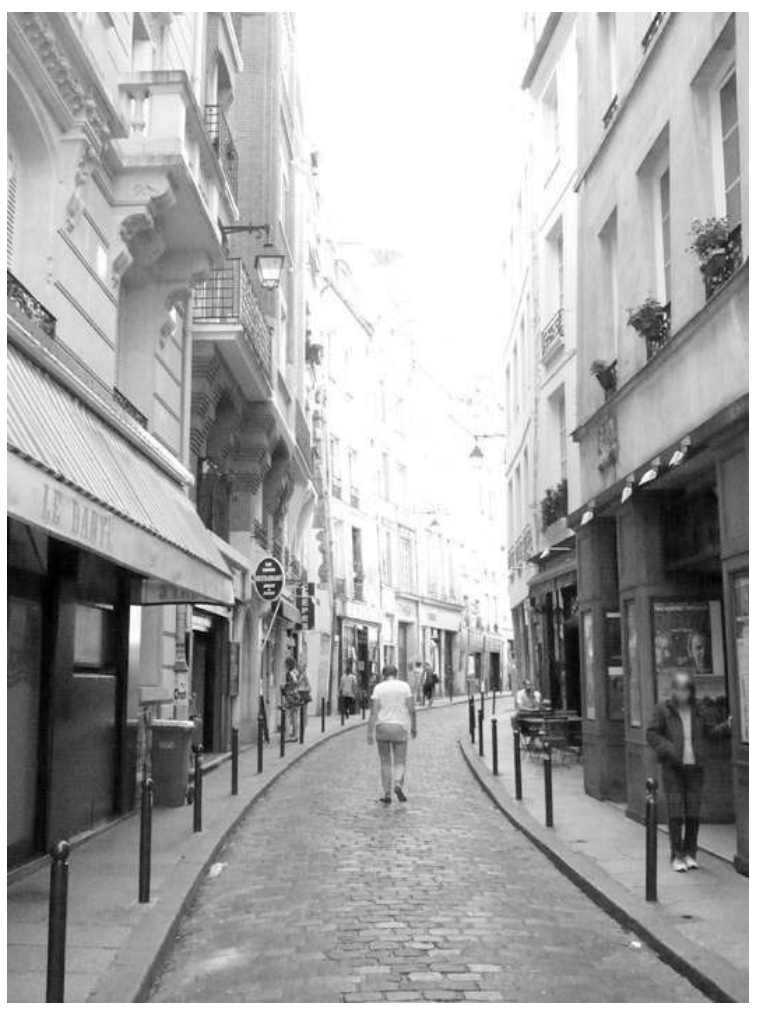

La rue Galande.

PHOT. HOTTIN, CHRISTIAN, 2014. (C) HOTTIN, CHRISTIAN.

Très vite attirés par le rayonnement de la jeune université, les dominicains et les franciscains s'établissent à Paris. Tout comme les séculiers, ils sont désireux de se tenir à distance du pouvoir épiscopal et cherchent à l'implanter sur la rive gauche. Un maître en théologie, Jean de Barastre, favorise la venue des dominicains en leur cédant une maison qu'il possède près de la porte Saint-Jacques : c'est bientôt là que s'élève leur couvent ${ }^{13}$. Les franciscains pour leur part trouvent un emplacement plus à l'ouest, de l'autre côté de la rue de la Harpe ${ }^{14}$ (fig. 3, 4). Ces deux établissements présentent la particularité de jouxter l'enceinte de Philippe Auguste : avec elle ils vont former de manière durable la limite occidentale du quartier des écoles. Quoique bâtis sans luxe, ces couvents vastes et très peuplés frappent l'imagination des contemporains, tel Guillaume de Saint-Amour, qui les qualifie de «Alta aedificia ${ }^{15}$. L'ampleur de ces locaux, ainsi que l'excellence et l'ambition des enseignants issus des ordres mendiants, contribue à attiser les rivalités entre ces derniers et les séculiers de l'Université. 
Figure 3

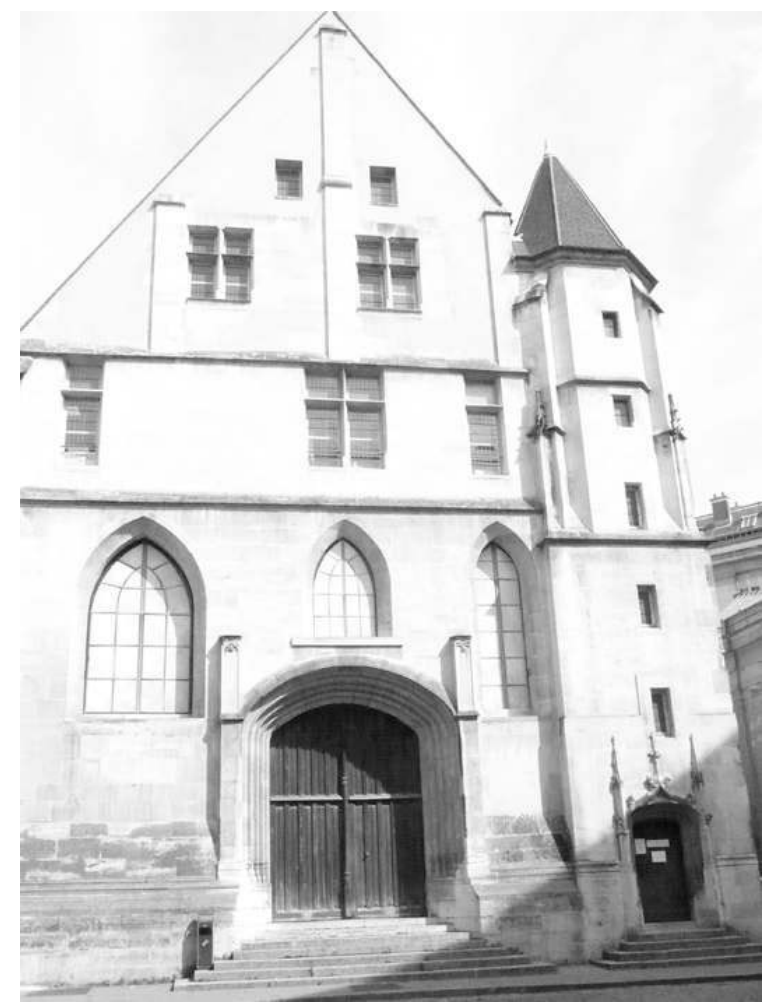

Le réfectoire des Cordeliers, actuellement inclus dans les bâtiments de l'Université Pierre et Marie Curie.

Phot. Hottin, Christian, 2014. (c) Hottin, Christian. 
Figure 4

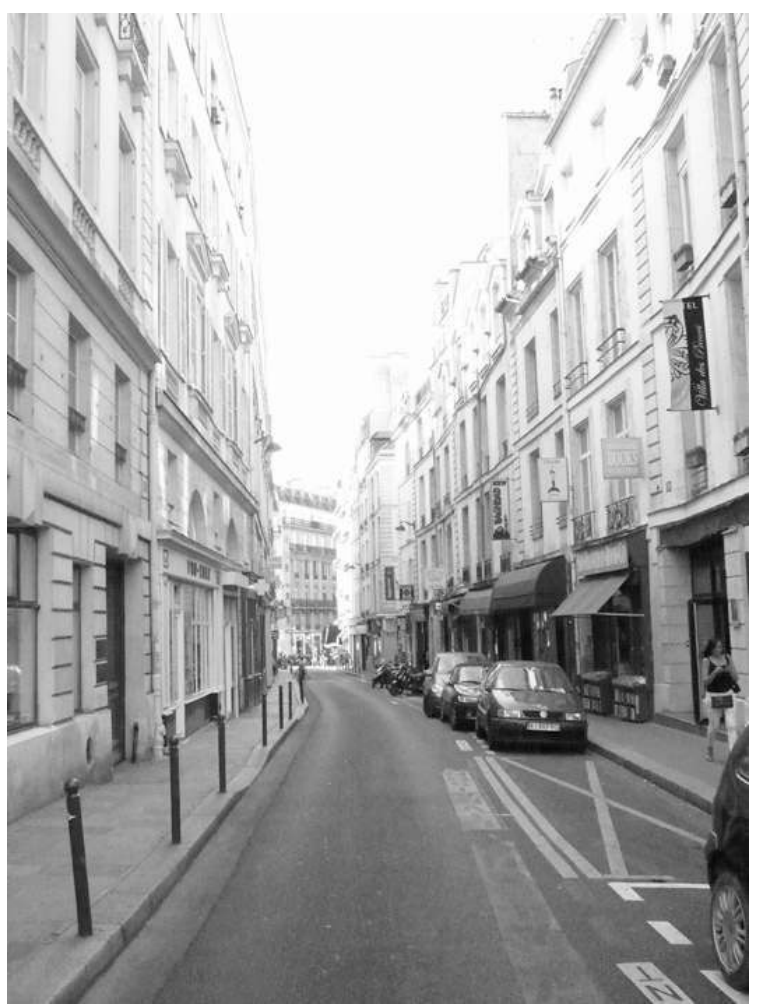

La rue Monsieur le Prince.

PHOT. HOTTIN, CHRISTIAN, 2014. @ HOTTIN, CHRISTIAN.

Vers la même époque, les collèges ${ }^{16}$, qui existaient depuis la fin du XII ${ }^{e}$ siècle $^{17}$ et se trouvaient parfois établis hors de la rive gauche ${ }^{18}$ deviennent de plus en plus nombreux et prennent place sur le flanc nord de la Montagne Sainte-Geneviève. Ainsi que l'a bien montré Aurélie Perrault ${ }^{19}$, à un premier mouvement de créations, essentiellement régulières, succède à partir des années 1250 un flux toujours croissant de fondations séculières. Il s'inscrit dans le prolongement de la naissance de la Sorbonne, autour de 1257. Douze fondations sont avérées entre 1250 et 1300, puis pas moins de 27 entre 1300 et 1350 , soit la moitié du nombre total de collèges de l'Université. Le mouvement se ralentit sensiblement par la suite, du fait de l'instabilité politique et des malheurs du temps ( 8 fondations entre 1350 et 1400,2 seulement après 1400 ). La fondation des collèges parisiens s'inscrit dans un mouvement général qui s'étend à toute l'Europe universitaire ${ }^{20}$ , mouvement qui persiste pendant toute l'époque moderne, même lorsque l'université entre en somnolence. Sans entrer dans le détail de l'architecture de ces établissements, il suffit de considérer que quelle que soit leur origine, tous sont désormais fondés au sud du fleuve. Il en va ainsi des fondations régulières : (fig. 5) Collège des Bernardins (1245, près de la rue Saint-Victor), Collège de Cluny (1262, en haut de la rue de la Harpe), Collège de Saint-Denis (1263, entre le quai des Augustins et la rue Saint-Germain) ou Collège des Prémontrés (1252, en face des Cordeliers) ${ }^{21}$. Les fondations séculières effectuées par des particuliers sont dans le même cas, qu'il s'agisse de celle de Robert de Sorbon ${ }^{22}$, du Collège d'Harcourt (1280, rue de Harpe ${ }^{23}$ ou de celui des Chollets (1290, rue Saint-Étienne des Grez $)^{24}$. Au tout début du siècle suivant, la fondation royale du Collège de Navarre et les hésitations sur sa localisation montrent bien que, non seulement la rive gauche apparaît comme le seul lieu possible pour l'installation d'un collège, mais encore que les 
fondateurs recherchent désormais des maisons et terrains dans une région bien précise, comprise entre la Seine et le sommet de la Montagne et structurée par de grandes voies de circulation orientées Nord-Sud (rue de la Harpe, rue Saint-Jacques, rue SainteGeneviève). Pour l'établissement de son collège, Jeanne de Navarre lègue son hôtel, situé près de la porte Saint-Germain, c'est-à-dire à l'ouest de la ville, et 2000 livres de rentes ${ }^{25}$. Un projet est étudié pour la transformation de la demeure royale en collège, mais Simon Festu, archidiacre de Vendôme qui est aussi l'un des huit exécuteurs testamentaires, convainc les sept autres d'abandonner ce projet et d'établir le nouveau collège "dans le quartier des écoles ». Le 12 avril 1309, la première pierre de la chapelle est posée ${ }^{26}$.

Figure 5

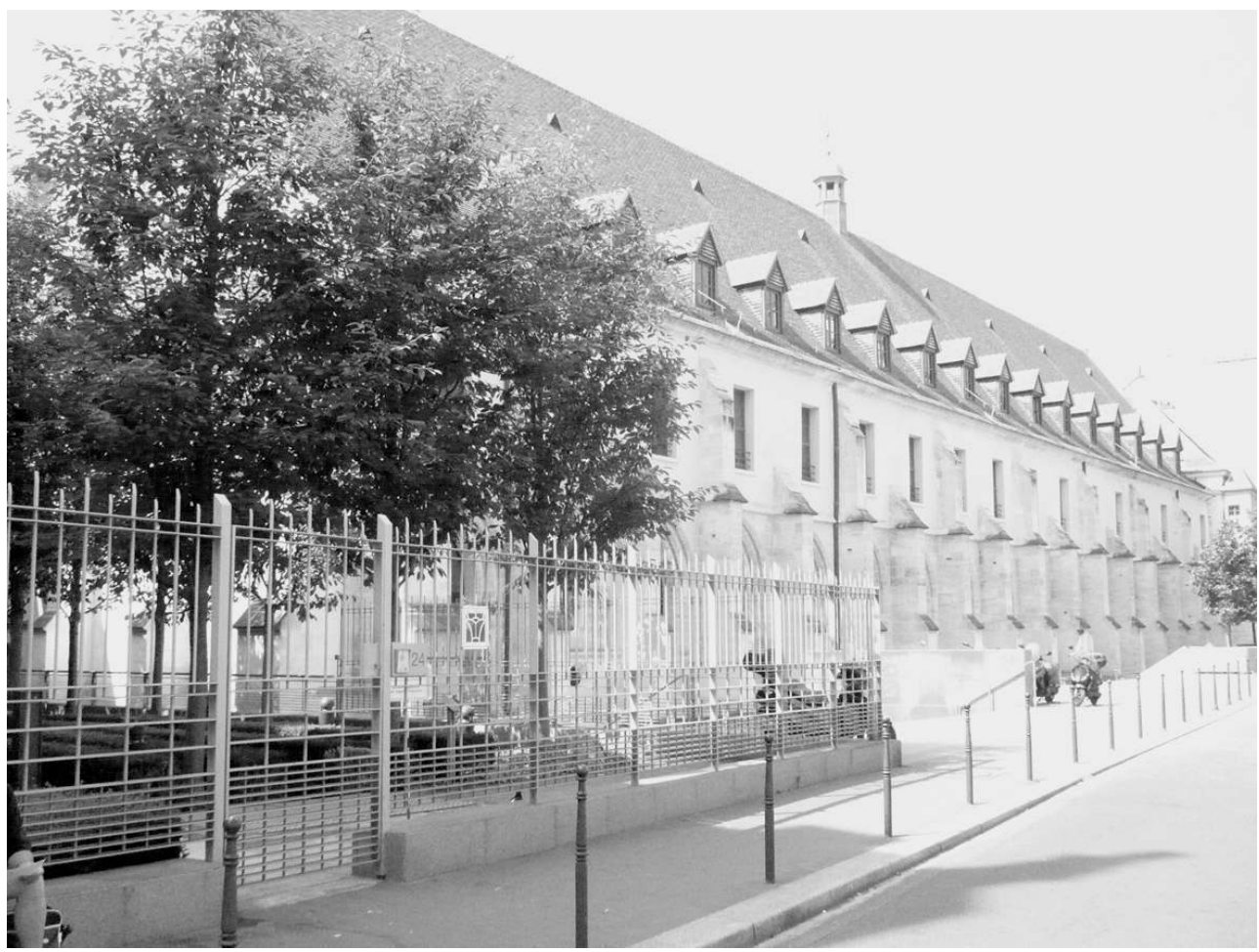

Le réfectoire des Bernardins, actuellement collège des Bernardins.

Phot. Hottin, Christian, 2014. (c) Hottin, Christian.

Le phénomène ici décrit de concentration des établissements liés à l'institution universitaire et contribuant à assurer sa visibilité alors même qu'elle est dépourvue d'édifices propres s'observe à la même époque dans les autres villes universitaires européennes $^{27}$. Dans le cas parisien, il convient en outre d'insister sur les importants pouvoirs de l'institution universitaire, grâce auxquels elle exerce de multiples manières une véritable autorité sur cette rive gauche qualifiée elle-même "d'Outre les ponts", «d'Outre le Petit Pont » ou tout simplement « d'Université ». En premier lieu, les métiers ayant trait à la fabrication des outils du travail intellectuel, tels que parcheminiers, relieurs, copistes, enlumineurs, libraires, et plus tard papetiers, sont soumis à son contrôle en tant que métiers «jurés» de l'Université. Ils versent lors de la prestation de serment une caution, et les litiges liés à leur pratique relèvent du tribunal du recteur ${ }^{28}$. C'est précisément par les droits de justice, alors les plus importants, que se manifeste le pouvoir de l'Université sur la rive gauche. Si elle n'est propriétaire d'aucun domaine censitaire à l'intérieur de l'enceinte, elle exerce des droits sur le Pré-aux-Clercs, vaste 
étendue non bâtie située à l'ouest de la ville et comprise entre l'enceinte de Philippe Auguste, la Seine et l'abbaye Saint-Germain-des-Prés. Cette dernière conteste longtemps l'autorité de l'Université sur ce territoire proche de ses propres bâtiments, mais les différents procès portés devant le parlement à ce sujet sont généralement gagnés par les universitaires ${ }^{29}$. Enfin, le droit de taxation des loyers, c'est-à-dire celui de fixer le prix des loyers dans les maisons habitées par des maitres ou des étudiants, fait partie des pouvoirs de l'Université. Déjà reconnu par les statuts de Robert de Courson en 1215, il est réaffirmé dans la bulle Parens scientiarum en 1231. Si aucune indication précise n'est donnée sur le territoire soumis à cette juridiction, divers documents, tels que les listes de taxations établies pour les années 1282 à 1289, font apparaître que presque toutes les maisons concernées se trouvent sur la rive gauche, quelques-unes seulement dans la Cité, et aucune sur l'autre rive. De même, le compte des bourses versées à l'Université par ses suppôts pendant la période 1329-1336 renferme quarante-sept noms de lieux, tous localisés sur la rive gauche. On y apprend en outre que tous les libraires, à une exception près, et tous les relieurs habitent alors « outre les ponts $»^{30}$. Enfin, des textes postérieurs invitent à penser que l'Université ne bénéficiait d'aucun droit sur la rive droite. Autant d'éléments qui invitent à épouser le point de vue de Serge Lusignan concernant l'existence d'un territoire universitaire parisien :

"Il semble donc que la jouissance des privilèges universitaires dépendît, non seulement de l'appartenance corporative, comme on l'a toujours su, mais aussi du fait d'habiter sur la rive gauche de la Seine, à l'intérieur de l'enceinte de Philippe Auguste, ou sur l'île de la Cité. Il existerait donc un véritable territoire de l'Université de Paris, dont les limites découlaient de l'histoire même des écoles parisiennes, nées autour du cloitre Notre-Dame, puis qui s'étaient déportées sur la rive gauche vers la montagne Sainte-Geneviève $»^{31}$.

Ce territoire, on peut tenter d'en dresser une première cartographie au bas Moyen Âge.

\section{Une première photographie, fin du XIV ${ }^{e}$ siècle, début de $\mathrm{XV}^{\mathrm{e}}$ siècle $^{32}$}

Alors que la ville s'est considérablement étendue sur la rive droite, au point d'être enserrée sous Charles $\mathrm{V}$ dans une nouvelle muraille, son développement au sud de la Seine demeure plus modeste, et l'agglomération reste pour l'essentiel contenue dans les limites de l'enceinte élevée sous Philippe Auguste.

Sur cette rive, la trame urbaine conserve quelques-uns des traits hérités de la Lutèce antique, notamment l'orientation Nord-Sud des principales voies de circulations, les deux plus importantes étant la rue de la Harpe et la rue Saint-Jacques. Comme partout ailleurs dans Paris, les églises sont nombreuses, et les plus importantes sont le siège des différentes paroisses de la rive gauche : Saint-André-des-Arts, Saint-Séverin, Saint-Cosme, Saint-Benoit-le-Bétourné, Saint-Hilaire, Saint-Étienne-du-Mont et Saint-Nicolas-duChardonnet. Ces églises, ainsi que de nombreuses chapelles, n'ont pas seulement une fonction strictement religieuse. À une époque où l'université et ses divisions (nations et facultés) n'ont guère de locaux propres, elles servent de lieu de rassemblement ou de dépôts pour leurs papiers ${ }^{33}$ : l'église des Mathurins est le théâtre de nombreuses assemblées et le point de départ des défilés et processions, la nation de France se réunit dans la chapelle des Bernardins ou à Saint-Nicolas-du-Chardonnet, celle de Picardie à Saint-Julien-le-Pauvre, celle d'Allemagne à Saint-Côme ou aux Jacobins ${ }^{34}$. Si tout le cœur du quartier est occupé par des immeubles, maisons et collèges, ses marges, en limite de l'enceinte du XIII siècle, le sont par de grands établissements religieux, couvents ou 
abbayes. D'ouest en est on rencontre : les Augustins, les Cordeliers, les Jacobins, l'Abbaye Sainte-Geneviève et les Bernardins. Ils sont plus rares vers le centre, mais trois sont groupés au pied de la colline, les Carmes, les Mathurins et la Commanderie des Hospitaliers de Saint-Jean de Jérusalem. Hors de l'enceinte, cette forte présence des réguliers est complétée vers l'ouest par la vieille Abbaye de Saint-Germain-des-Prés et vers l'est par celle de Saint-Victor.

Depuis le XIII siècle, le nombre des collèges n'a cessé de croître, et ce mouvement va perdurer jusqu'au XV siècle: 11 fondations avant 1300, 27 de 1300 à 1350, 8 de 1350 à 1400,2 encore après $1400^{35}$. Ils sont présents partout sur la rive gauche, mais la plus forte concentration se trouve sur le flanc nord-ouest de la Montagne, dans la partie exactement comprise entre le pied de la côte et son sommet. Vers l'ouest, de part et d'autre de la rue de la Harpe, sont groupées les fondations les plus anciennes : (fig. 6) la Sorbonne (1257), le Collège de Rethel, ceux de Cluny (1262), du Trésorier (1268), de Calvi (1271), d'Harcourt (1280), de Bayeux (1309) et de Narbonne (1317). Plus bas dans la rue se trouvent des établissements moins anciens, les collèges de Justice (1353), de Dainville (1380), de Séez (1437). Aurélie Perrault, mêlant étude chronologique et approche topographique, a clairement montré que le choix d'installer à la Sorbonne à proximité immédiates des grands établissements mendiants (Cordeliers et Jacobins), avait été éminemment politique: il s'agissait de contrer leur influence sans cesse croissante. Cette volonté déboucha sur un renforcement de la présence des collèges dans ce secteur, la rue de la Harpe devenant l'axe principal de leur implantation ${ }^{36}$.

Figure 6

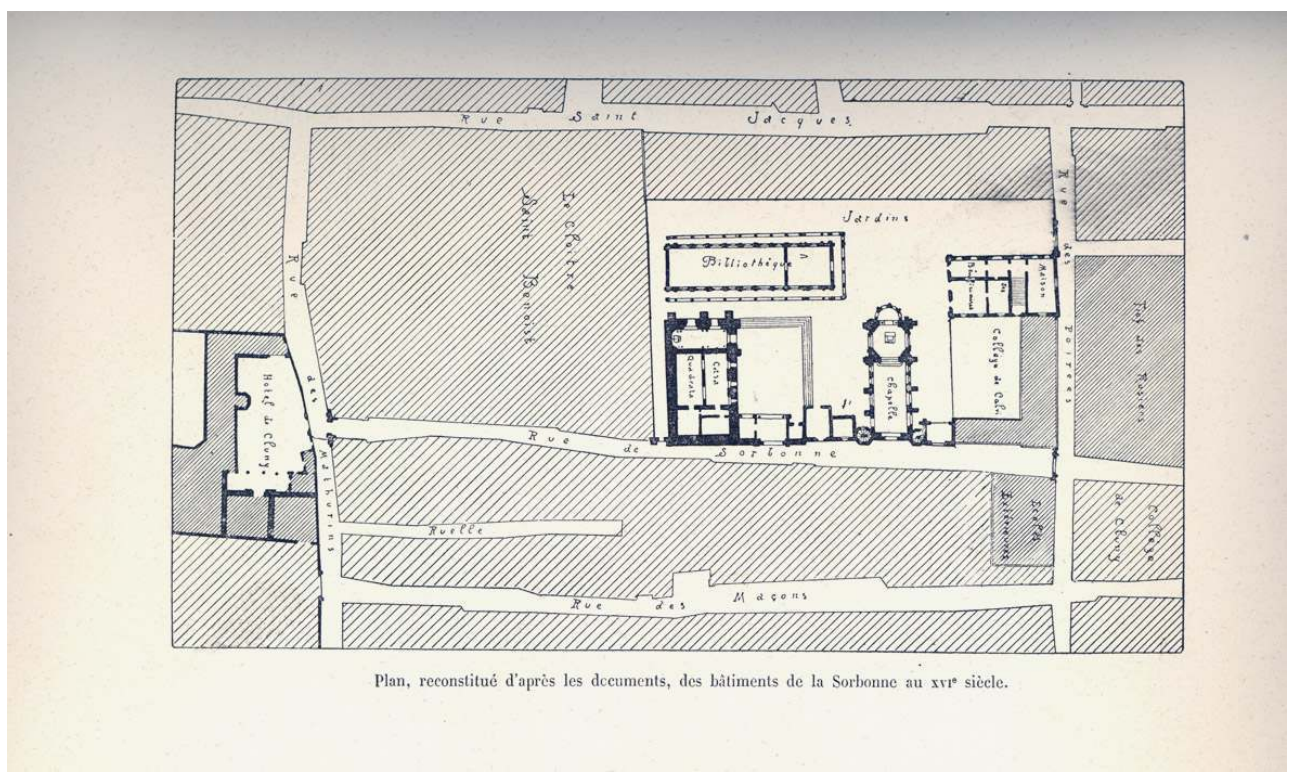

Plan restitué de la Sorbonne à l'époque médiévale. Source : GREARD, Octave. Nos adieux à la vieille Sorbonne. Paris : Hachette, 1893, p. 85.

Le second groupe de collèges est borné par les rues des Noyers, Sainte-Geneviève, du Moustier, Saint-Étienne des Grez et Saint-Jacques. Il s'agit pour l'essentiel de fondations du XIV ${ }^{e}$ siècle, postérieures donc au groupe occidental. La plus ancienne est le Collège des Chollets (1282), suivi par ceux de Montaigu et de Presles (1314). Viennent ensuite les Collèges du Plessis (1322), de Tréguier (1325), de Huban (1327), de Marmoutiers (1329), des Lombards (1333), de Laon (1339), de Cambrai (1344), de Beauvais (fig. 7) (1370), de 
Fortet (1394), de Reims (1412) et de Lisieux (1414). Non loin de là, sur la rue SainteGeneviève et son prolongement formé par la rue de la Porte Bordelle, sont regroupées d'autres créations datant du XIV ${ }^{\mathrm{e}}$ siècle : le Collège de Navarre (1304), séparé de ceux de Boncourt et Tournai (1353) par l'étroite rue Clopin. Pour cet ensemble, Aurélie Perraut a mis en évidence le rôle joué par le couvent des Carmes et par le collège de Navarre, véritables "pôles d'attraction et de développement pour les autres fondations ", selon une répartition géographique qui privilégie encore l'axe nord-sud: soit la rue SaintJacques, soit les rues qui montent de la place Maubert ${ }^{37}$.

Figure 7

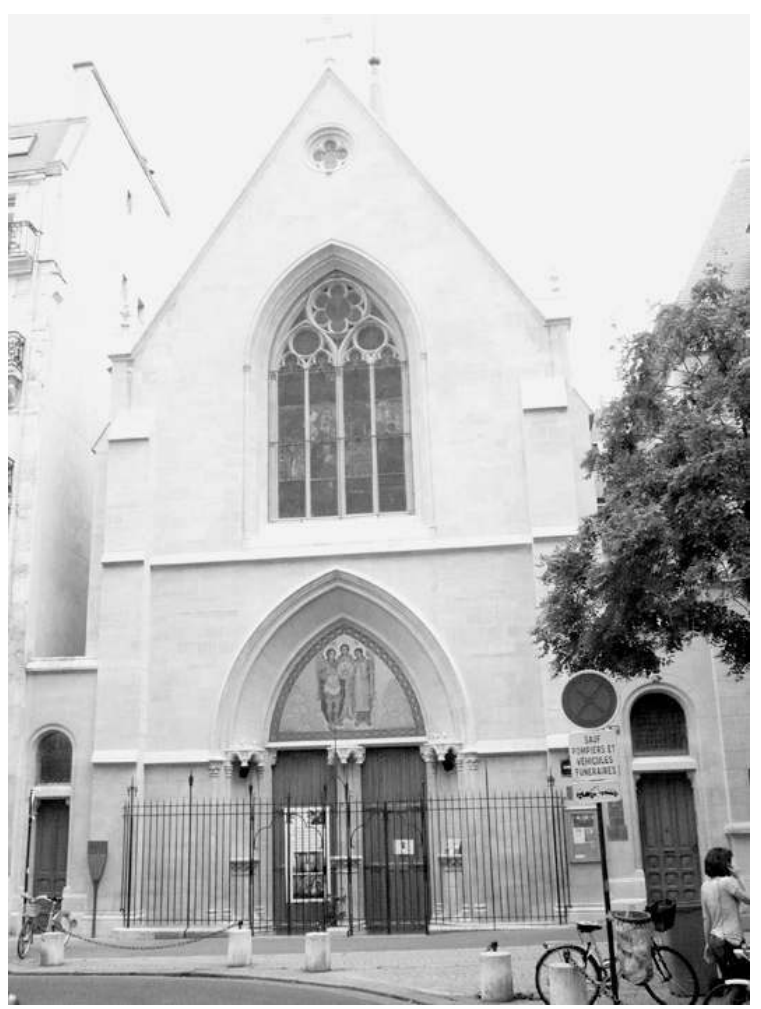

La chapelle du collège de Beauvais, actuellement église orthodoxe roumaine des Saints-Archanges.

Phot. Hottin, Christian, 2014. (c) Hottin, Christian.

Toute la partie basse de la rive gauche, comprise entre le fleuve et le piémont de la colline, accueille également des collèges. Certains sont anciens, antérieurs ou contemporains du phénomène de concentration issu de l'installation de la Sorbonne: Collège des Bons Enfants (1250), Écoles de Saint-Denis (1263), Collège des Prémontrés (1252), collège du Cardinal Lemoine (1302). Les onze autres datent tous du XIVe siècle et sont implantés sans former de regroupement (Bourgogne, Autun, Boissy, Mignon, Tours, Maitre Gervais, Cornouailles, Chanac, Picardie, Arras et de la Marche). On trouve encore des collèges, souvent anciens, sur la rive droite (des Bons enfants - 1208 - et de SaintNicolas), ou dans la Cité (des Dix-huit, crée en 1180), où les écoles cathédrales sont actives jusqu'au XIV ${ }^{\mathrm{e}}$ siècle.

Les lieux d'enseignement liés aux différentes facultés sont répartis un peu partout dans le quartier, au sein des collèges (théologie en Sorbonne et Navarre) ou à proximité : les écoles de Décret rue Jean de Beauvais au sud du collège de cette ville, la Faculté des arts 
rue du Fouarre. À la fin du XV ${ }^{e}$ siècle, l'École de Médecine prend ses quartiers non loin de là, rue de la Bûcherie (fig. 8).

Figure 8

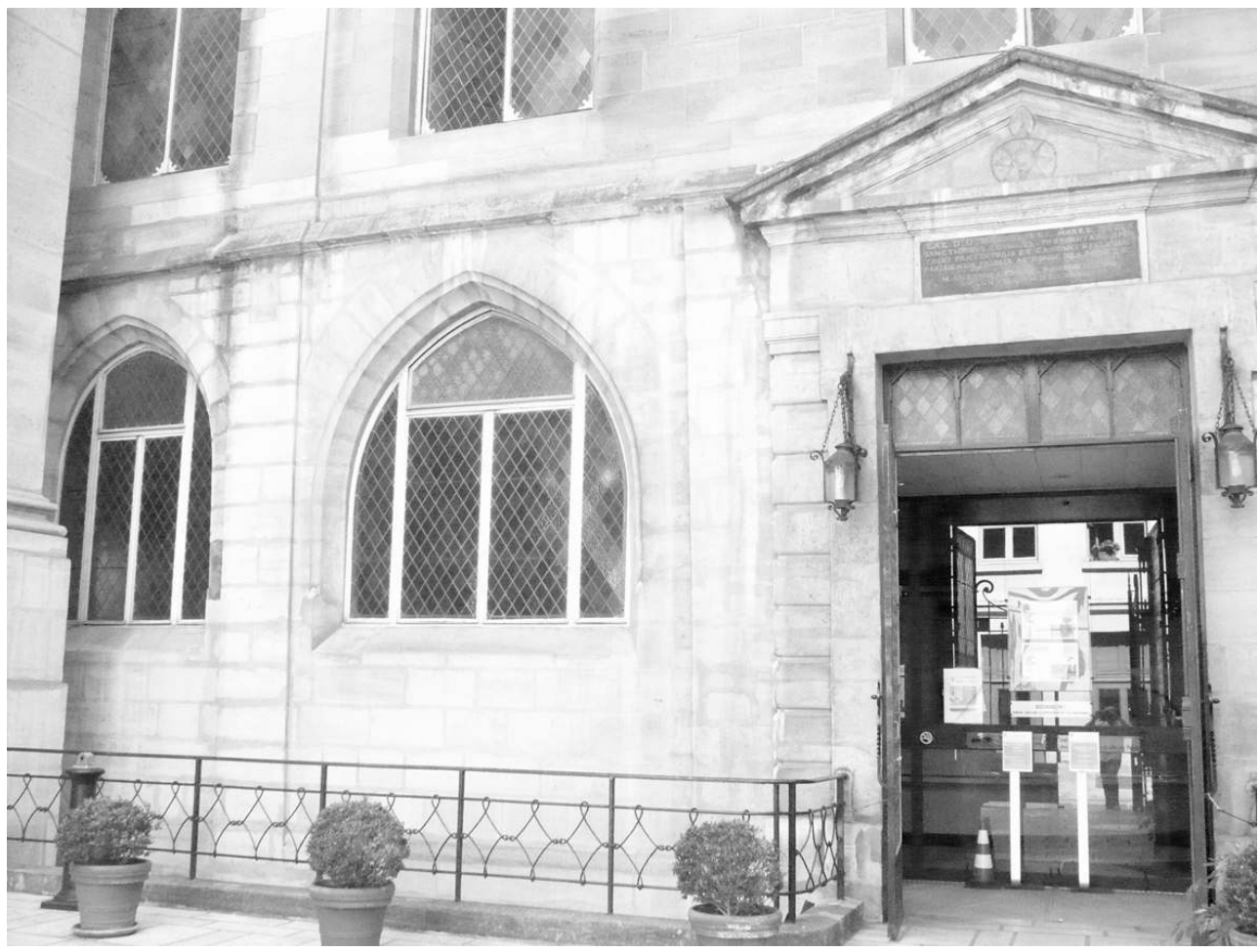

Les vestiges de l'ancienne Faculté de médecine, actuellement services de la Ville de Paris.

Phot. Hottin, Christian, 2014. (c) Hottin, Christian.

La comparaison entre la date de fondation des établissements et leur localisation géographique met en évidence trois phénomènes consécutifs. Dans un premier temps (avant les années 1250), on constate une relative dispersion des fondations, qui se font principalement mais non exclusivement au sud du fleuve, et plutôt dans la partie basse du quartier. Dans un deuxième temps (depuis les années 1250 jusqu'à la fin du XIII ${ }^{\mathrm{e}}$ siècle), un certain nombre de créations sont décidées dans le secteur compris entre les rues de la Harpe et Saint-Jacques (avec un dépassement de celle-ci pour les collèges des Chollets et de Montaigu) : cette concentration survient après la création de la Sorbonne, qui est avec le collège d'Harcourt le principal établissement de cette région. Enfin, tout au long du XIV e siècle, on assiste à un grand nombre de créations à l'est de la rue Saint-Jacques, tandis que reprennent les implantations de collèges - souvent de petite taille - dans une zone proche du fleuve.

\section{L'époque moderne : de l'enracinement à l'immobilisme}

(fig. 9) 


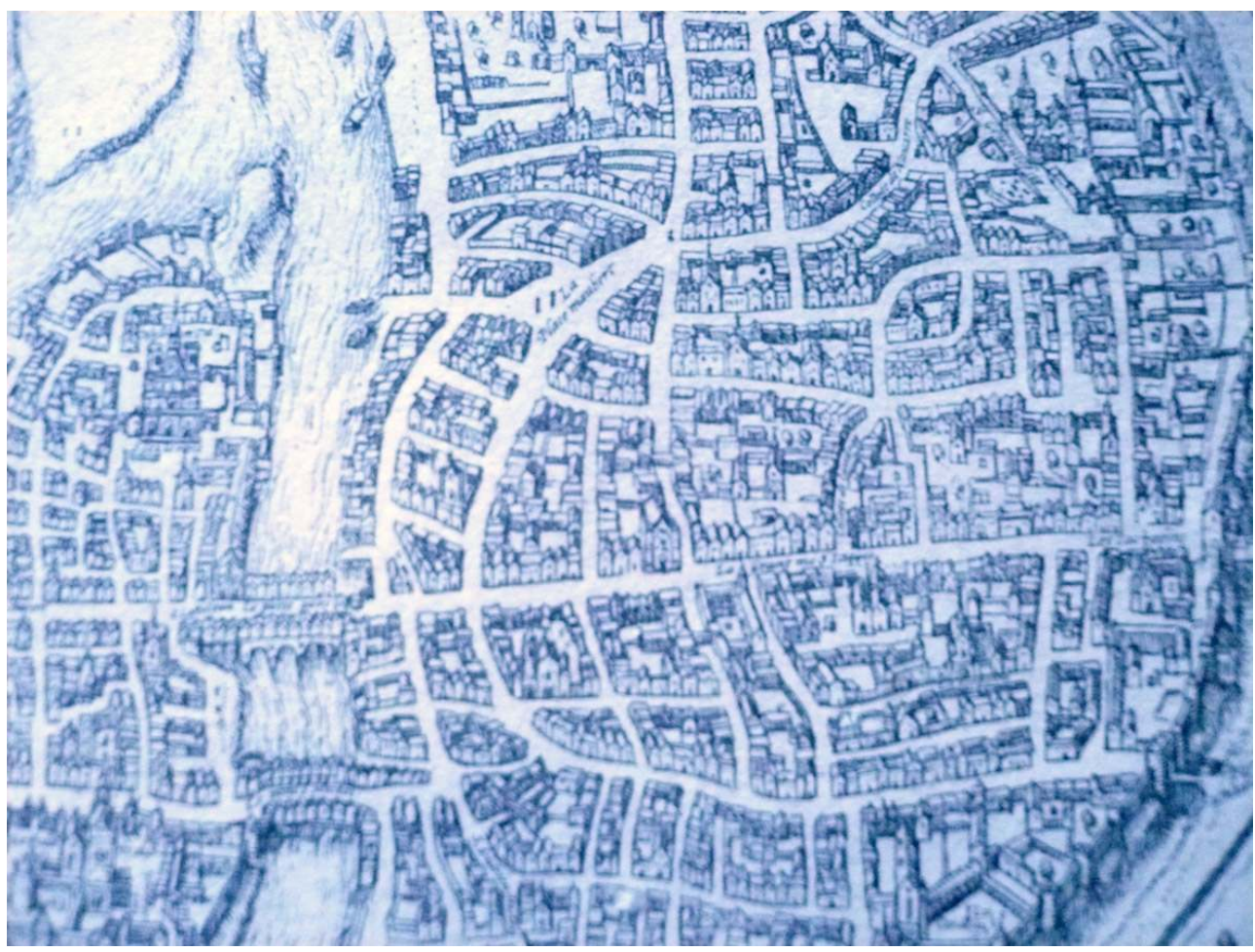

Le quartier de l'Université dans le plan de Truchet et Hoyaux, dit de Bâle (1550). Source : Bibliothèque nationale de France, cartes et plans. (C) BnF.

23 À la fin du Moyen Âge, l'importance des collèges dans l'organisation globale de l'enseignement ne cesse de croître, et les facultés doivent bientôt se résoudre à n'être, par le biais des examens et de la collation des grades, que des institutions de validation des connaissances acquises au sein d'autres établissements ${ }^{38}$. À la même époque, ces collèges voient leur rôle conforté par l'importance accordée au contrôle de la population estudiantine: la résidence au sein d'un collège devient une obligation pour tous les étudiants, à l'exception des nobles. À Paris, la réforme du cardinal d'Estouteville (1452) et le nouveau statut de la Faculté des Arts (1457) enjoignent aux artiens libres, ou martinets, de prendre logement dans une " pédagogie ", avec interdiction d'en changer au cours des études $^{39}$. L'enseignement des arts se transporte alors de la rue du Fouarre vers les collèges, au sein desquels se développent bibliothèques et salles de cours magistrau ${ }^{40}$.

Dans la capitale, cette prééminence du collège comme lieu de vie commune et d'enseignement va de pair avec la recherche d'une localisation dans le quartier qui est, dès cette époque, perçu comme étant historiquement celui des écoles.

\section{$X V I^{e}$ et $X V I^{e}$ siècles : nouvelles fondations et anciens locauX}

(fig. 10, 11) 
Figure 10

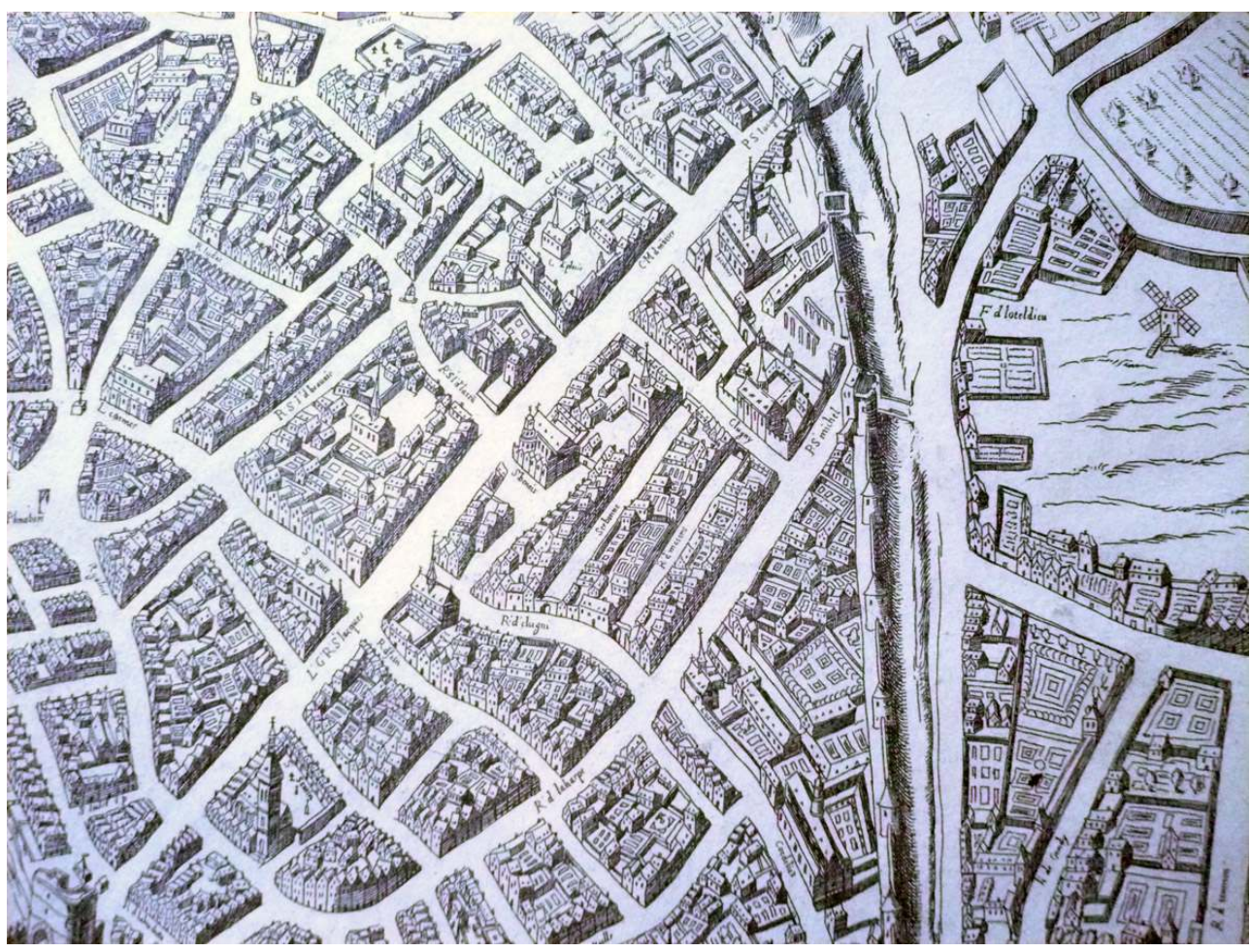

Le quartier de l'Université dans le plan de François Quesnel (1609). Source : Bibliothèque nationale de France, cartes et plans.

(C) BnF. 
Figure 11

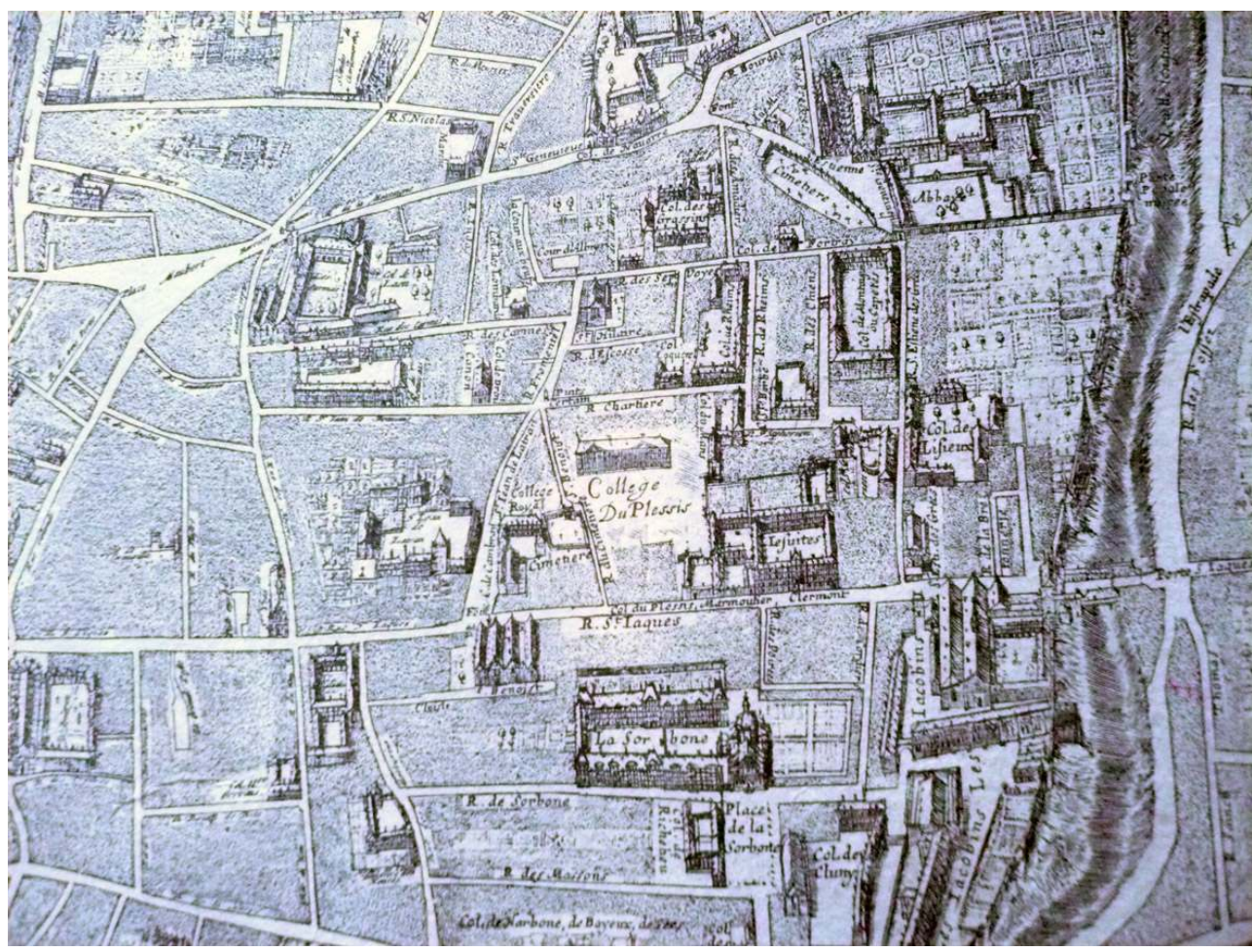

Le quartier de l'Université dans le Gomboust (1652). Source : Bibliothèque nationale de France, cartes et plans.

(C) BnF.

L'histoire géographique de la venue à Paris des fils de Saint Ignace témoigne, comme deux cent cinquante ans plus tôt la fondation du Collège de Navarre, de l'importance que revêt pour les fondateurs ou utilisateurs d'un établissement d'enseignement une implantation sur la rive gauche. Les jésuites fréquentent dans un premier temps le Collège du Trésorier puis le Collège des Lombards, avant d'être accueillis par le chancelier Duprat dans l'hôtel de Clermont, sis rue de la Harpe ${ }^{41}$. Cette solution provoque la colère du chapitre de Clermont, car la demeure n'appartient pas à Duprat mais au diocèse et est en principe réservée aux seuls clercs auvergnats séjournant à Paris. Peu après, grâce à l'argent légué par Duprat, augmenté des donations reçues des mains de membres du parti catholique, ils font l'acquisition de la cour de Langres, dans la rue Saint-Jacques. Cet hôtel était précédemment le séjour des évêques de Langres à Paris, et sa configuration convient bien mieux que l'hôtel de Clermont au projet d'installation des jésuites. En dépit des difficultés qui leur sont faites, ces derniers prennent possession des lieux en $1563^{42}$. Peu d'années auparavant, lorsque l'institution des lecteurs royaux trouve sous le nom de Collège Royal un local permanent, c'est non loin du futur séjour des jésuites, dans les bâtiments des collèges mitoyens de Tréguier et de Cambrai ${ }^{43}$. 
Figure 12

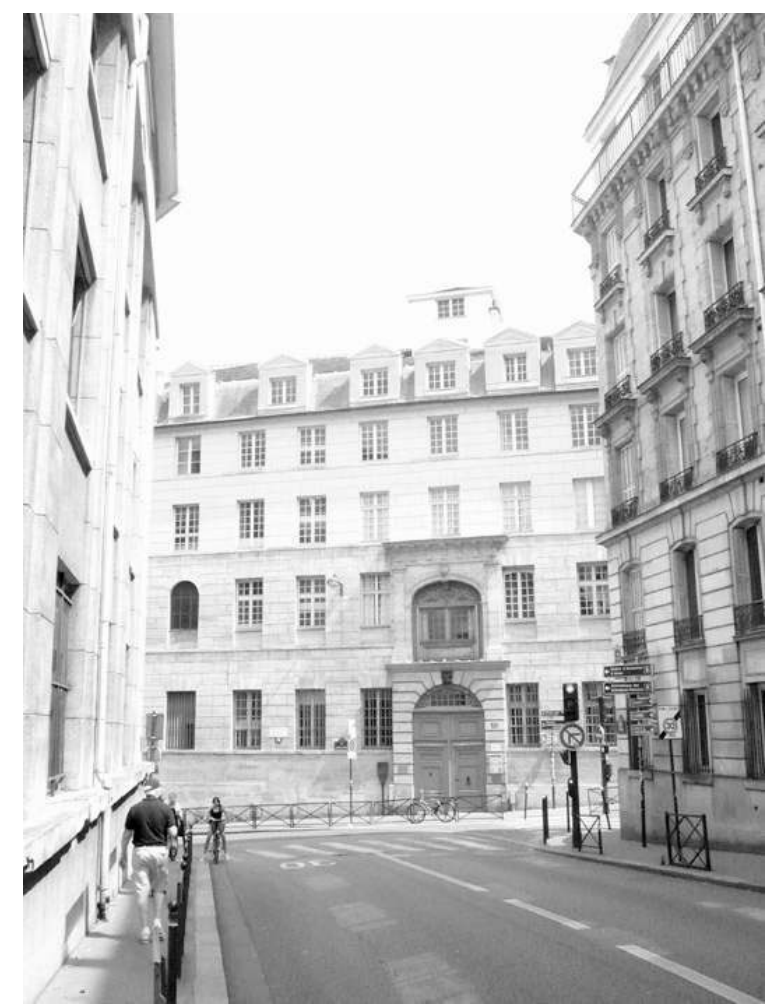

Le collège des Écossais, actuellement école primaire et foyer d'étudiantes.

Phot. Hottin, Christian, 2014. (c) Hottin, Christian.

Sous Henri IV est fondé le Collège des Écossais (fig. 12). Là encore, toute la géographie de la nouvelle institution s'organise autour de la Montagne Sainte-Geneviève. En 1603, l'archevêque de Glasgow et ambassadeur d'Écosse en France, James Beaton, lègue sa maison de la rue des Amandiers (actuelle rue Laplace) aux artiens et théologiens de son pays qui étudiaient à Paris. En 1639 ce collège fusionne avec l'ancien Collège d'Écosse, fondé au XIV siècle par l'évêque de Murray, et qui se trouvait près du Collège de Cambrai. Enfin en 1665, les deux établissements réunis gagnent la rue des fossés SaintVictor (actuelle rue du Cardinal Lemoine) où un nouveau local est élevé pour eux ${ }^{44}$. Peu après est établi le Séminaire et Collège des Irlandais : Louis XIV lui concède dans un premier temps les locaux de l'ancien collège des Lombards, depuis longtemps désaffecté ${ }^{45}$, mais en 1769 la communauté fait l'acquisition d'un nouveau local, sis rue du cheval vert (actuelle rue des Irlandais), qui est agrandi par François-Joseph Bellanger entre 1772 et 1775 (fig. 13). 
Figure 13

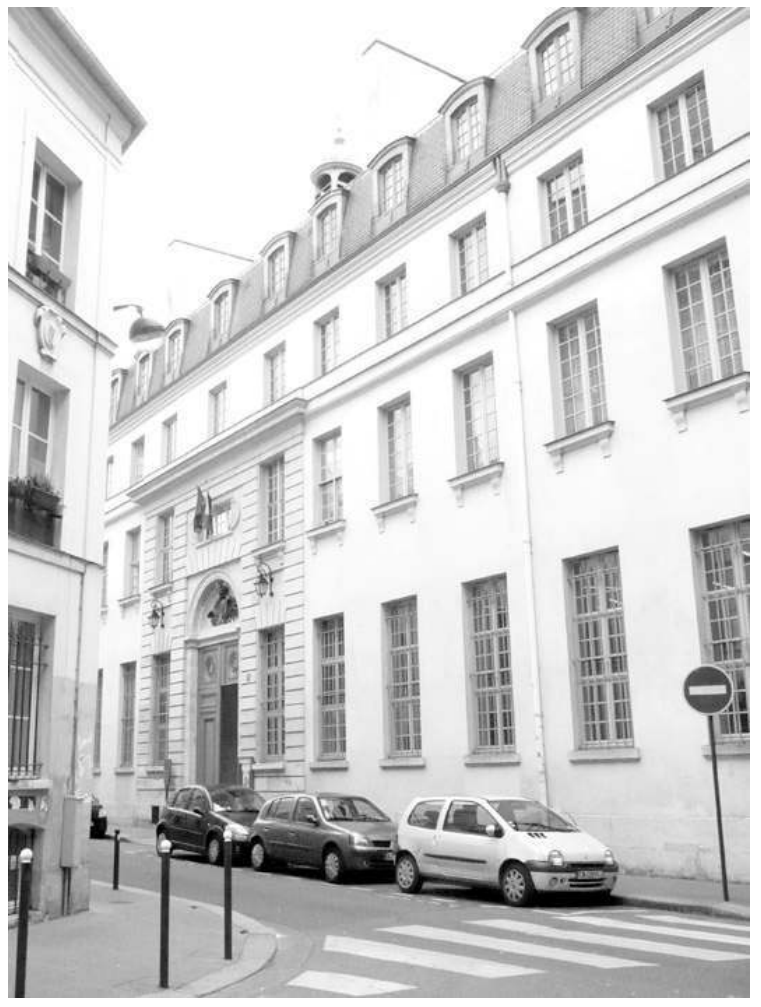

Le collège des Irlandais, actuellement centre culturel irlandais Phot. Hottin, Christian, 2014. (c) Hottin, Christian. 
Figure 14

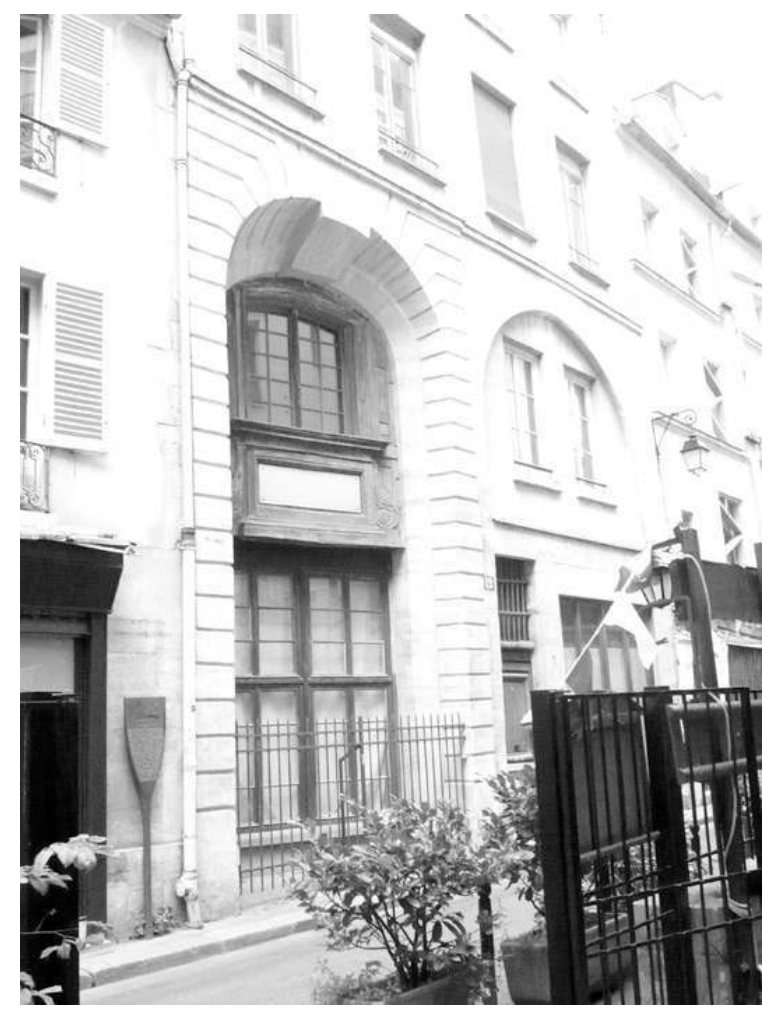

L'ancienne entrée du collège des Grassins, actuellement maison particulière.

Phot. Hottin, Christian, 2014. (c) Hottin, Christian.

Ces fondations nouvelles, comme on le voit, prennent parfois la place de collèges tombés en décadence et dont les locaux sont désaffectés. D'autres créations sont quant à elles établies dans des locaux neufs, tel le Collège des Grassins (fondé par Pierre de Grassin en 1569) (fig. 14) ou le Collège des Quatre-Nations (fig. 15), voulu par Mazarin, agrégé à l'université et achevé en 1688. Mais l'un comme l'autre s'élèvent sur la rive gauche, qu'il s'agisse de l'étroite rue des Amandiers, au pied de l'Abbaye Sainte-Geneviève ou des quais de la Seine, face au Louvre ${ }^{46}$. Par delà les transformations institutionnelles s'affirme aux $\mathrm{XVI}^{e}$ et $\mathrm{XVII}^{e}$ siècles une stabilité géographique qui va devenir source de problèmes au siècle suivant (fig. 16). 


\section{Figure 15}

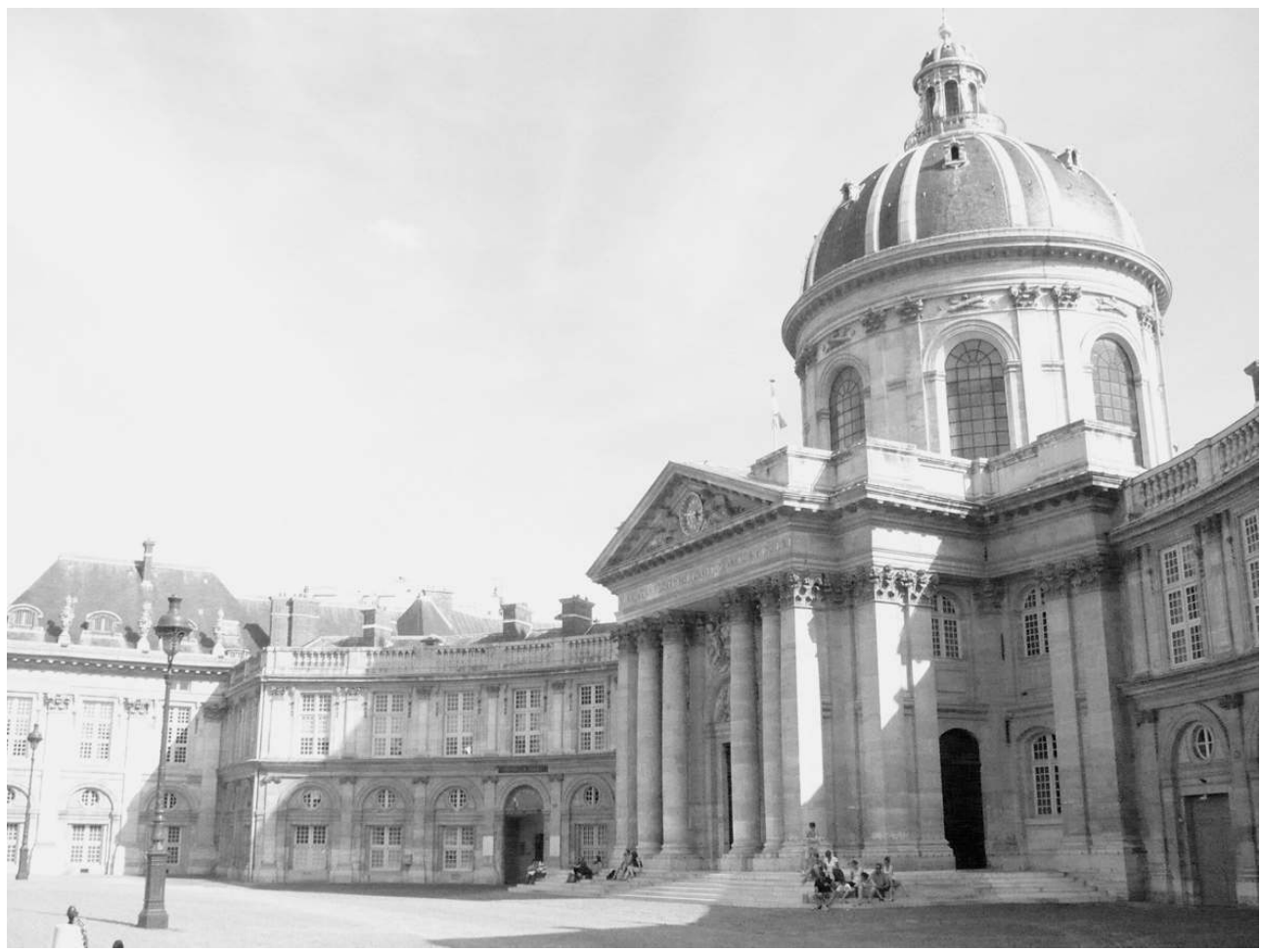

Le collège des Quatre-Nations, actuellement Institut de France.

Phot. Hottin, Christian, 2014. (c) Hottin, Christian. 


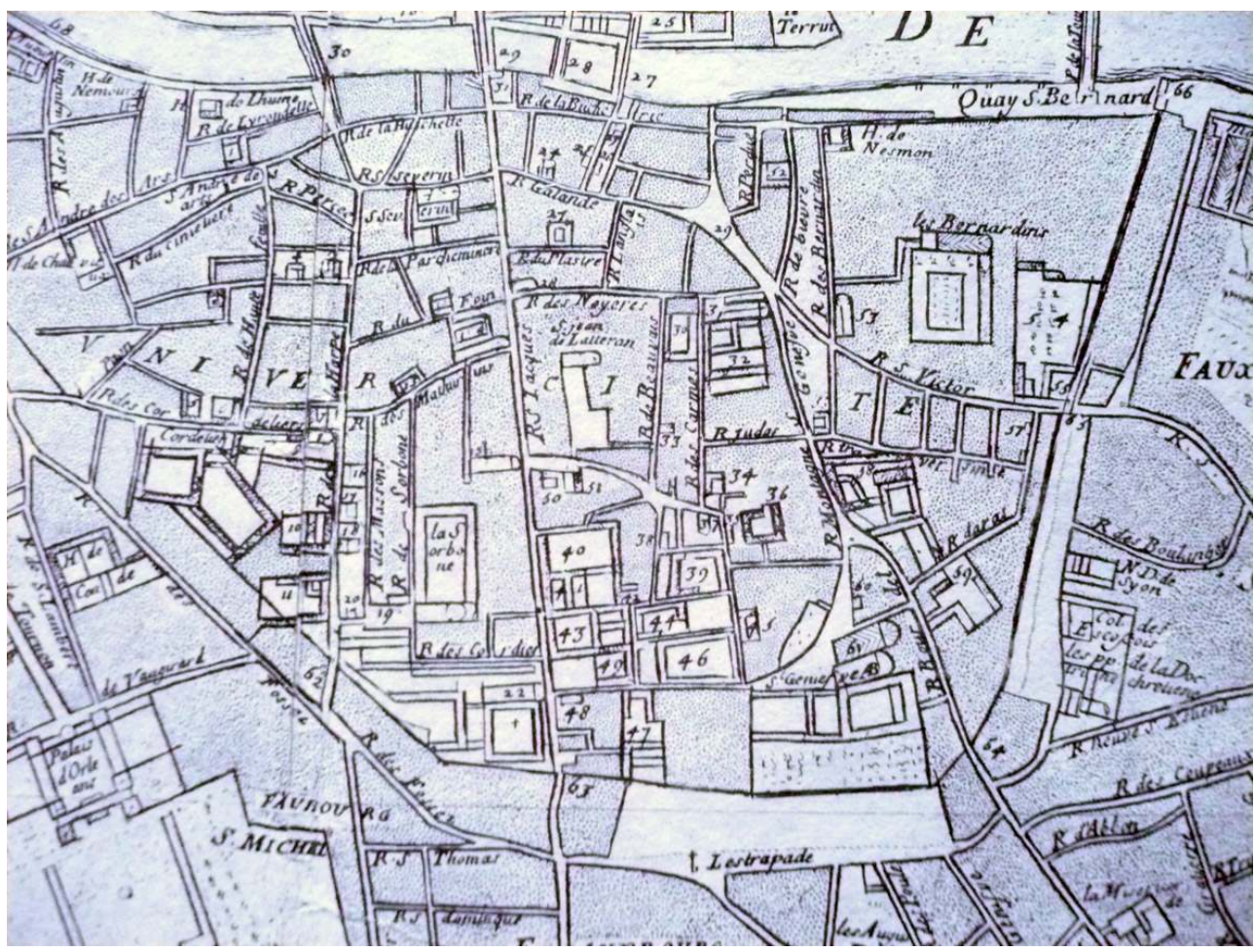

Le quartier de l'Université dans le plan de Nicolas le Fer d'après Jouvin de Rochefort (1676). Source: Bibliothèque nationale de France, cartes et plans.

(C) BnF.

\section{Au XVIIII siècle : la suppression des jésuites et des petits collèges, une chance manquée de visibilité nouvelle pour l'Université}

À partir de la Régence, la forte concentration des collèges sur la Montagne SainteGeneviève apparait comme un handicap plus que comme un atout. En effet, le riche établissement des jésuites, désormais dénommé Collège Louis-le-Grand, mène une rude concurrence aux fondations de l'Université, qui se trouvent finalement trop nombreuses pour le quartier. Le nouveau recteur, Petit de Montempuis, propose en 1715 une réforme de la Faculté des arts qui prévoit, outre l'instauration de la gratuité des études dans cette faculté, le transfert de certains collèges - on cite ceux des Grassins, de Lisieux et de Montaigu - sur la rive droite, où les établissements scolaires sont rares. Petit de Montempuis se heurte à de vives oppositions et doit renoncer à cet aspect de sa réforme ${ }^{47}$ (fig. 17). 


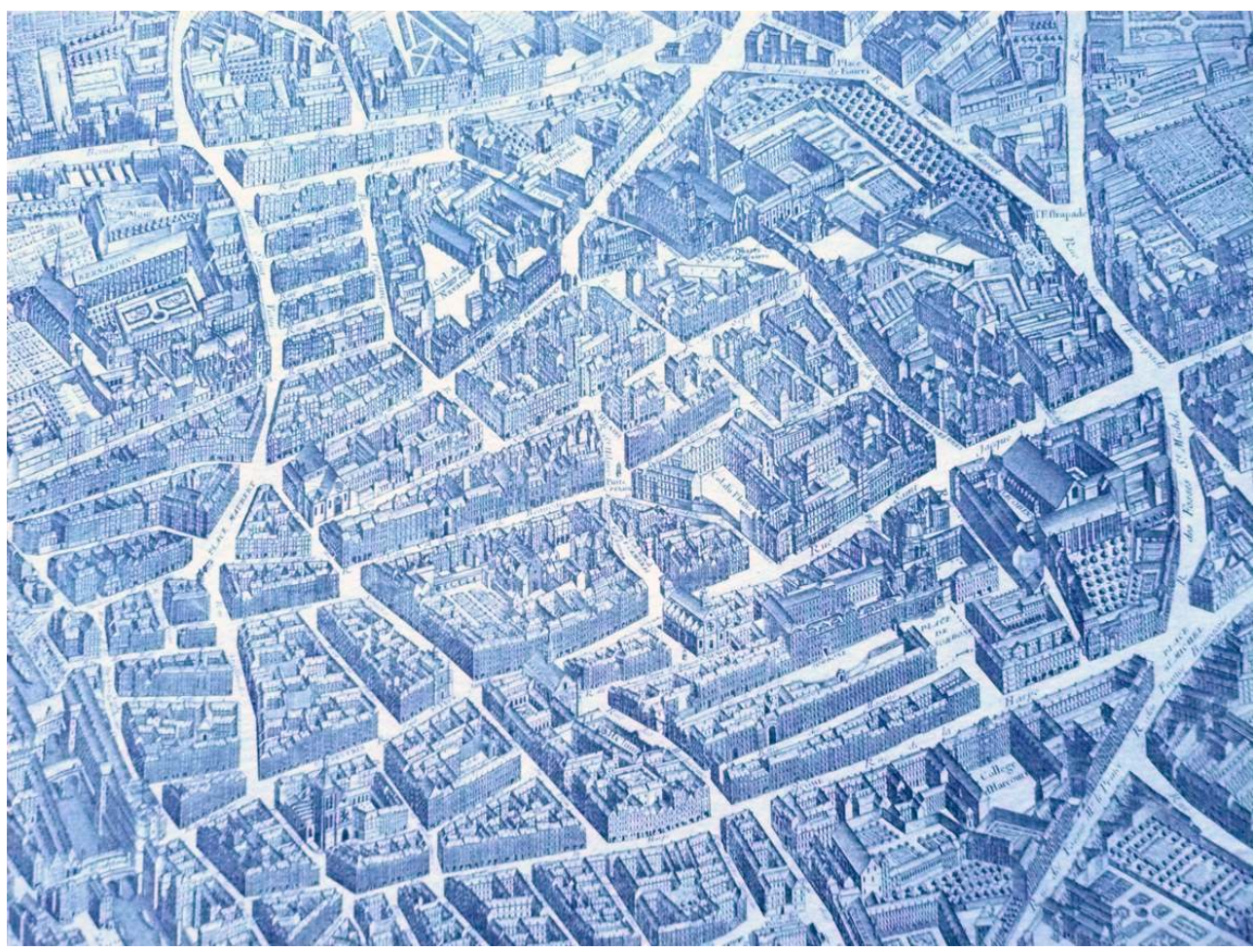

Le quartier de l'Université dans le plan de Turgot (1734-1739). Source : Bibliothèque nationale de France, cartes et plans.

(C) BnF.

Si elle ne résout pas tous les problèmes géographiques de l'université, la suppression des jésuites va néanmoins causer des bouleversements considérables, qui donnent à l'Université de Paris, à partir de 1762, une physionomie sensiblement différente de celle qui était la sienne depuis les temps médiévaux. L'arrêt du Parlement du 6 août 1762 qui supprime la Compagnie de Jésus vient après la création du Concours général (1747) mais avant celle de l'agrégation (1766) et prend place dans un processus de transformation de l'institution universitaire qui affecte également sa vie matérielle. Les locaux du Collège Louis-le-Grand étant devenus vacants, le Parlement décide le 7 septembre 1762 d'y transférer le Collège de Lisieux, dont les anciens bâtiments doivent être détruits pour faire place à la nouvelle église Sainte-Geneviève. On ordonne surtout, conformément aux vœux des enseignants, de réunir dans le vaste édifice tous les boursiers des petits collèges de la Montagne ${ }^{48}$. Si les bourses qui étaient attachées aux fondations sont conservées, les établissements n'en disparaissent pas moins en tant que bâtiments spécifiques. Vingt-sept institutions sont ainsi supprimées. Il est vrai, cependant, que beaucoup n'avaient plus vers 1750 qu'une existence purement nominale ${ }^{49}$ (fig. 18). Dès 1764, à la demande de l'évêque de Lisieux et de l'archevêque de Reims, abbé de Fécamp, qui protestent contre l'installation du collège de Lisieux dans les bâtiments jésuites, on procède à un échange : le Collège de Dormans-Beauvais s'installe dans les locaux de Louis-le-Grand et celui de Lisieux dans ceux de Dormans-Beauvais. C'est à cette époque que l'Université établit son siège à Louis-le-Grand et que sa bibliothèque nouvellement créée y prend ses quartiers ${ }^{50}$. 
Figure 18

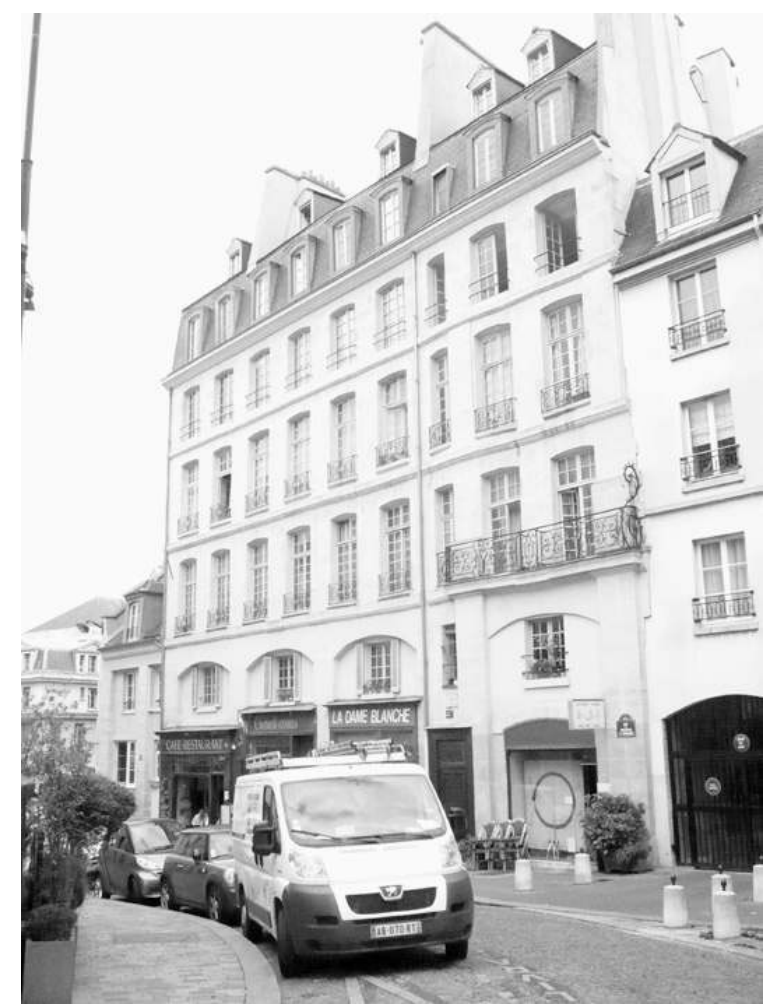

Le collège de Huban ou de l'Ave Maria, actuellement maison particulière.

Phot. Hottin, Christian, 2014. (c) Hottin, Christian. rationalisation de l'espace universitaire, pourrait apparaître comme le premier acte d'une rénovation de l'Université. Il n'en a rien été. Il est même frappant de constater que les dernières décennies de la Monarchie, marquées par la construction ou la translation de nombreux établissements, facultés ou écoles, sont pour l'Université celles des échecs et des projets avortés ou rejetés.

En effet, depuis le départ des jésuites, l'Université voit la population de ses collèges augmenter. Elle atteindra 5000 étudiants en 1789. Pour désengorger le quartier Latin, plusieurs propositions de déménagements sont évoquées, qui toutes échouent du fait du refus des universitaires. C'est d'abord la requête du principal du Collège de Lisieux, qui juge son établissement trop à l'étroit rue Jean de Beauvais et souhaite que lui soient attribués les bâtiments de la maison professe des jésuites, située sur la rive droite (fig. 19) , dans un quartier dépourvu de tout collège ${ }^{51}$. L'université refuse d'accéder à sa demande, craignant qu'un collège implanté sur l'autre rive n'échappe finalement à son contrôle. Quelques années plus tard, le prince Philippe de Poix, gouverneur de Versailles, suggère à Louis XVI de transférer à Versailles un des collèges de Paris. Là encore, l'Université refuse toute installation en dehors du quartier des écoles ${ }^{52}$. Rétive aux propositions qui lui sont faites, elle échoue dans la promotion de ses propres projets : alors qu'elle désire pour la première fois de son histoire établir son siège dans un bâtiment spécifique, en l'occurrence un édifice de même apparence que la nouvelle Faculté de Droit de Soufflot et lui faisant face au sud de la façade principale de Sainte-Geneviève, elle doit renoncer à cette ambition en raison du piètre état des finances publiques ${ }^{53}$. 
Figure 19

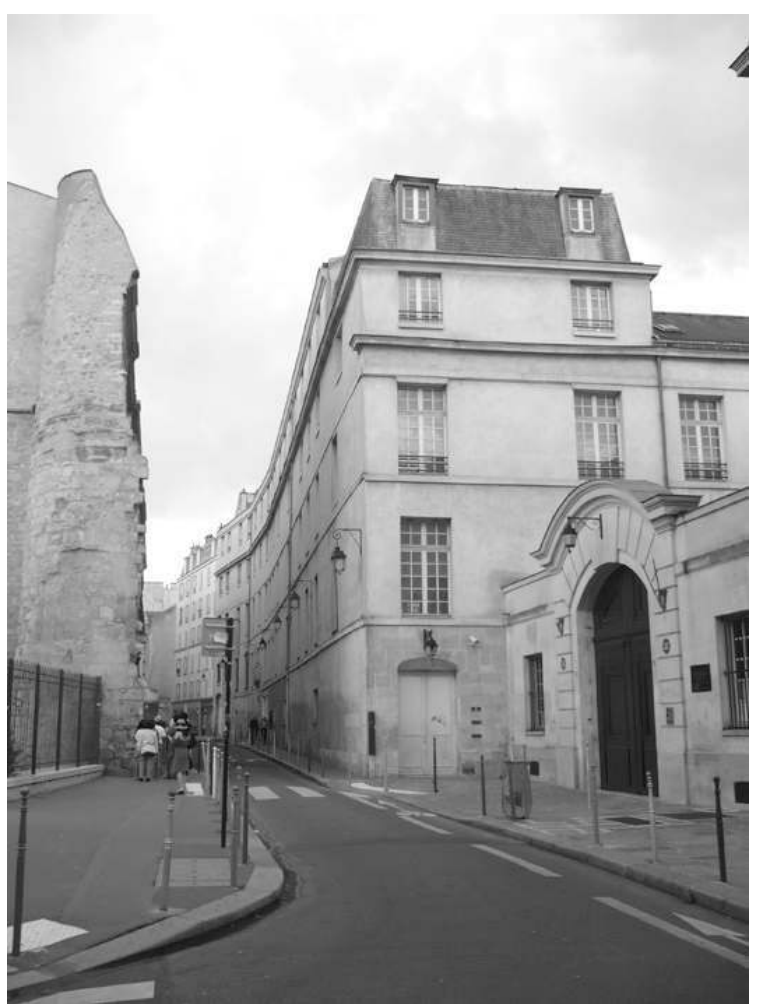

La maison professe des Jésuites, actuellement lycée Charlemagne.

Phot. Hottin, Christian, 2014. (c) Hottin, Christian.

Les projets couronnés de succès sont pourtant nombreux dans ces années. Entre 1763 et 1772 est réalisée la translation des Écoles de Droit du Clos-Bruneau au sommet de la Montagne Sainte-Geneviève, dans le bâtiment conçu par Soufflot (fig. 20). La Faculté de Médecine, à l'étroit dans sa vieille maison de la rue de la Bûcherie, en profite pour prendre possession des anciens locaux des juristes ${ }^{54}$. À l'ouest du quartier Latin, les chirurgiens abandonnent leur amphithéâtre voisin de l'église Saint-Côme (fig. 21) pour prendre leurs aises dans le vaste édifice (fig. 22) que Gondoin leur a élevé à l'emplacement du Collège de Bourgogne, dont les terrains avaient été acquis par le roi à cette fin dès $1769^{55}$. 


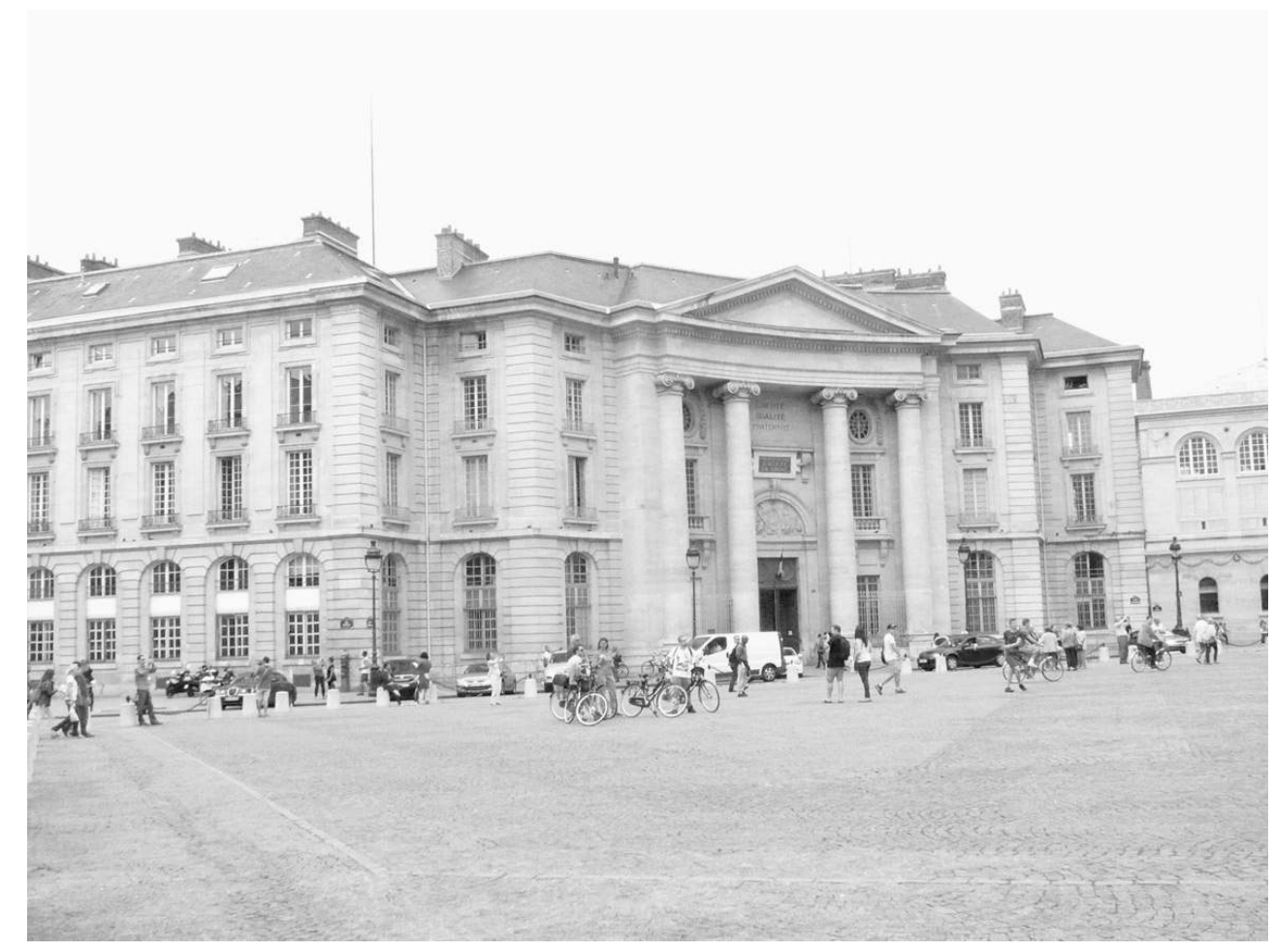

La Faculté de droit, actuellement Universités Paris 1 Panthéon-Sorbonne et Paris 2 Panthéon-Assas. Phot. Hottin, Christian, 2014. (c) Hottin, Christian. 
Figure 21

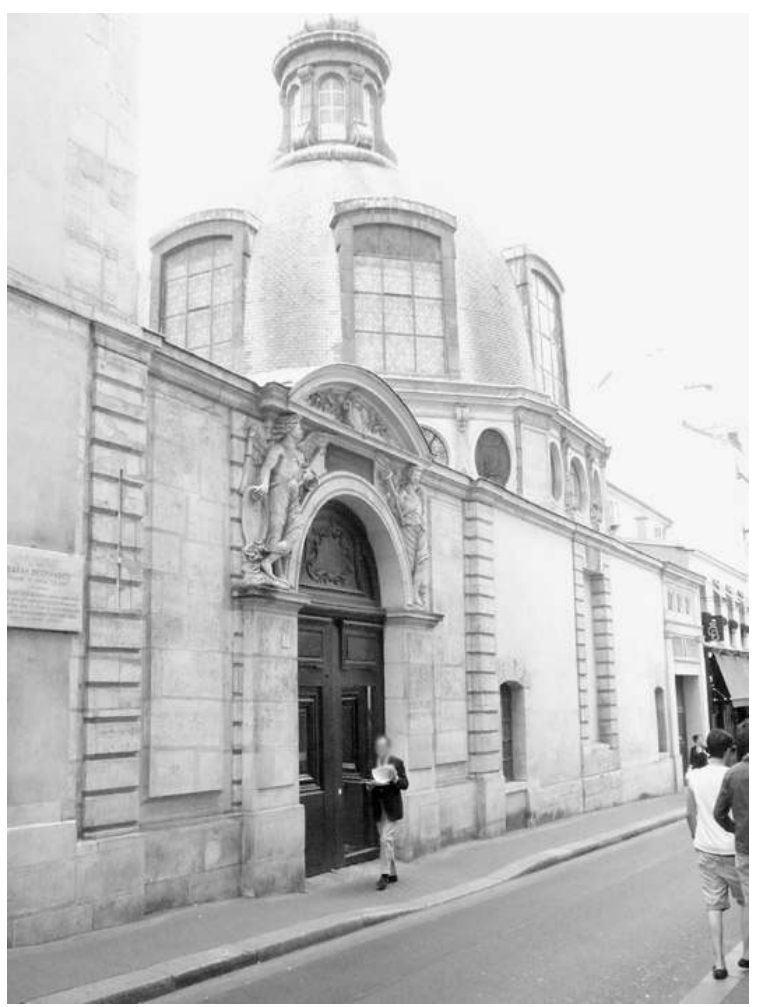

L'amphithéâtre de Saint-Cosme du collège de chirurgie, actuellement Université Paris 3 Sorbonne nouvelle.

Phot. Hottin, Christian, 2014. (c) Hottin, Christian. 


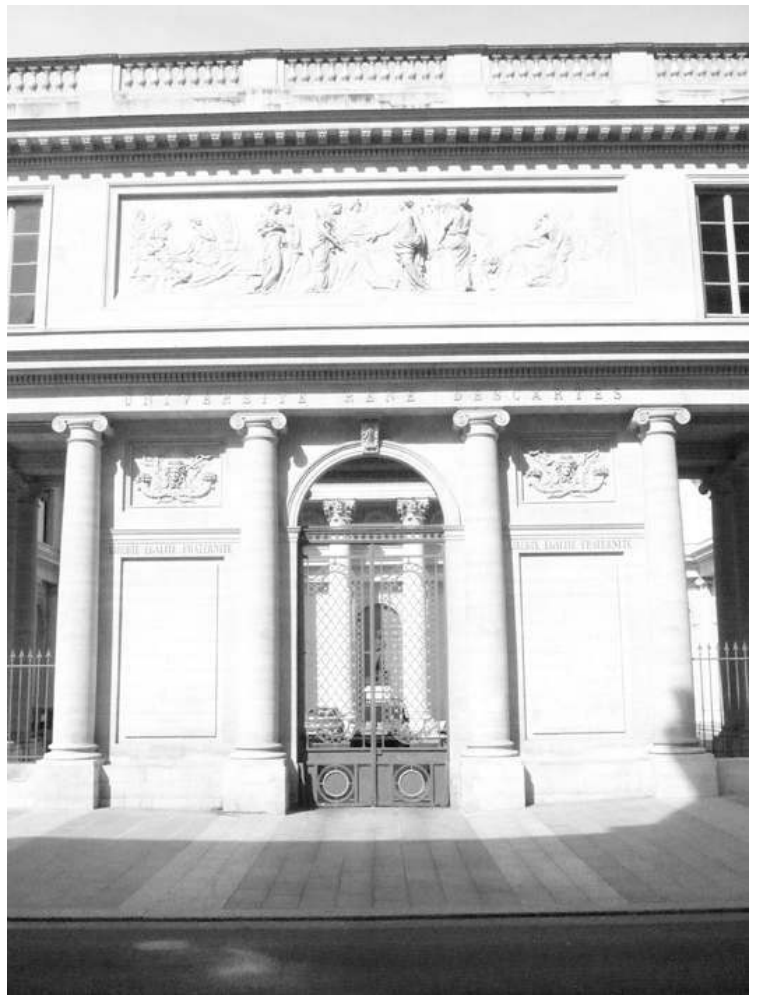

L'Académie de chirurgie, actuellement Université Paris 5 René Descartes. Phot. Hottin, Christian, 2014. (c) Hottin, Christian.

Leur ancienne demeure ne reste pas inoccupée bien longtemps, puisque Bachelier y transporte son École gratuite de dessin. Enfin, en 1773, le Collège Royal est réuni à l'Université, malgré les réticences de cette dernière, et l'abbé Terray, Contrôleur général des finances, affecte des sommes considérables à sa reconstruction par Chalgrin (fig. 23). La potion est pour l'université d'autant plus amère que les sommes en question, produit des revenus de ses messageries, auraient dû être réservées à la construction de son nouveau siège ${ }^{56}$. À titre de compensation, Terray a auparavant proposé sans succès à l'Université de prendre ses quartiers dans le collège désaffecté des Chollets. 


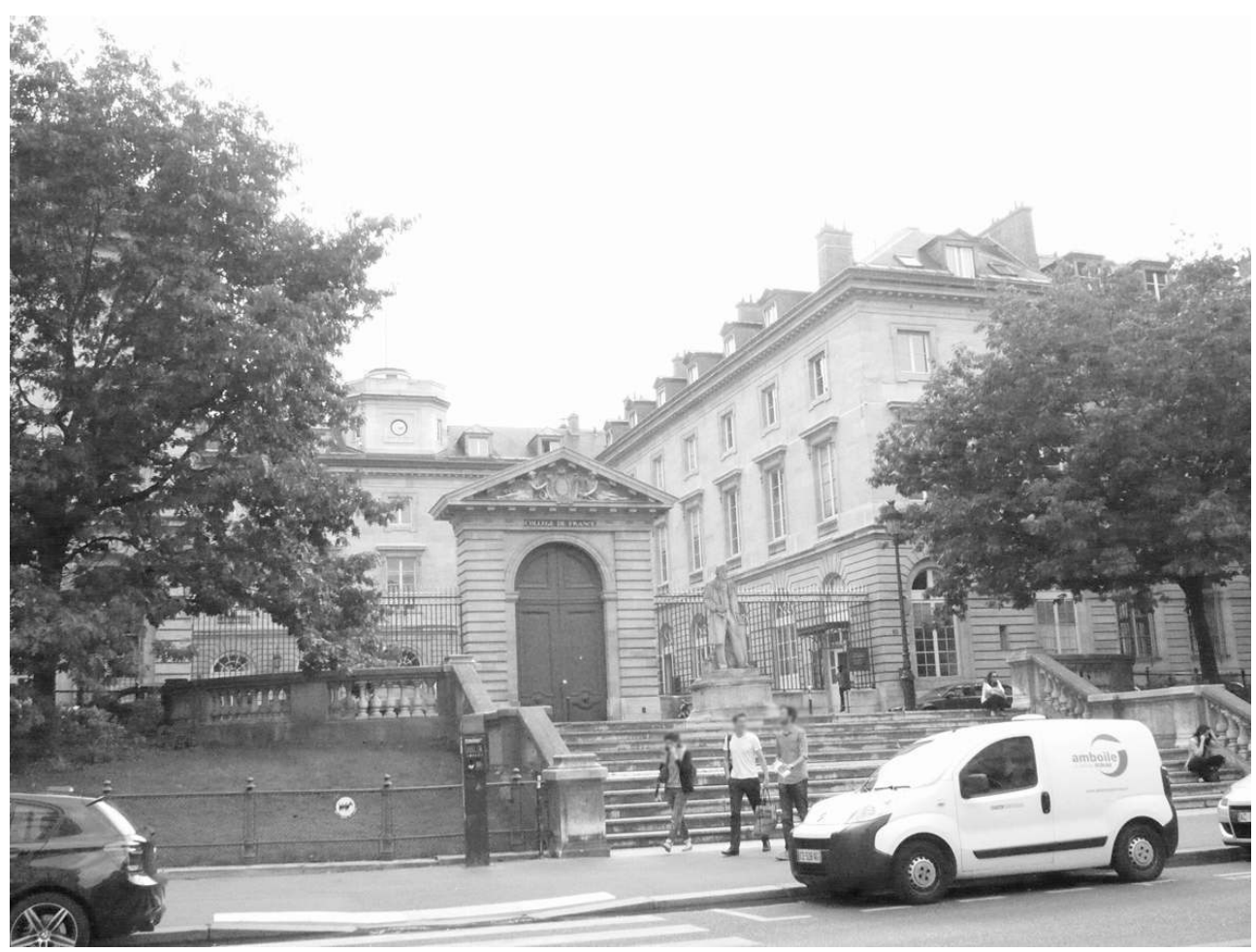

Le Collège de France.

PHOT. HOTTIN, CHRISTIAN, 2014. ㄷ HOTTIN, CHRISTIAN.

Les décisions consécutives au départ des jésuites, si elles ont modifié la topographie universitaire du quartier Latin, n'ont pas suffi à insuffler un esprit de réforme durable : incapable de se réformer en tant que corps, l'université est en 1789 également inapte à projeter son action sur un territoire autre que celui qui est le sien depuis le XIII ${ }^{\mathrm{e}}$ siècle. Elle demeure attachée à cet espace historiquement constitué, alors même que les besoins de l'éducation et le souci naissant d'aménagement rationnel de l'espace urbain appellent une tout autre répartition des lieux d'enseignement au sein des villes. La répartition des collèges parisiens est du reste vivement critiquée par Laugier dans ses Observations sur l'architecture :

«C'est contre toute raison que dans Paris les collèges se trouvent entassés dans un seul et unique quartier. Il en résulte pour le plus grand nombre des citoyens un éloignement qui rend la fréquentation de ces collèges impraticable. Chaque quartier devrait avoir le sien, et cet arrangement pourrait se faire sans dépense, en prenant pour cela dans chaque quartier une des maisons religieuses qui s'y rencontre et en transportant la communauté dans quelqu'un des anciens collèges (...) S'il n'y a qu'un seul collège dans une ville, on doit le placer vers le centre et dans un endroit d'où les communications multipliées bannissent les embarras. S'il y en a plusieurs, ils doivent être distribués dans les différents quartiers de la ville pour la commodité des citoyens $»^{57}$.

\section{Le plan de Verniquet ${ }^{58}$ : une deuxième photographie ${ }^{59}$}

(fig. 24, 25, 26) 
Figure 24

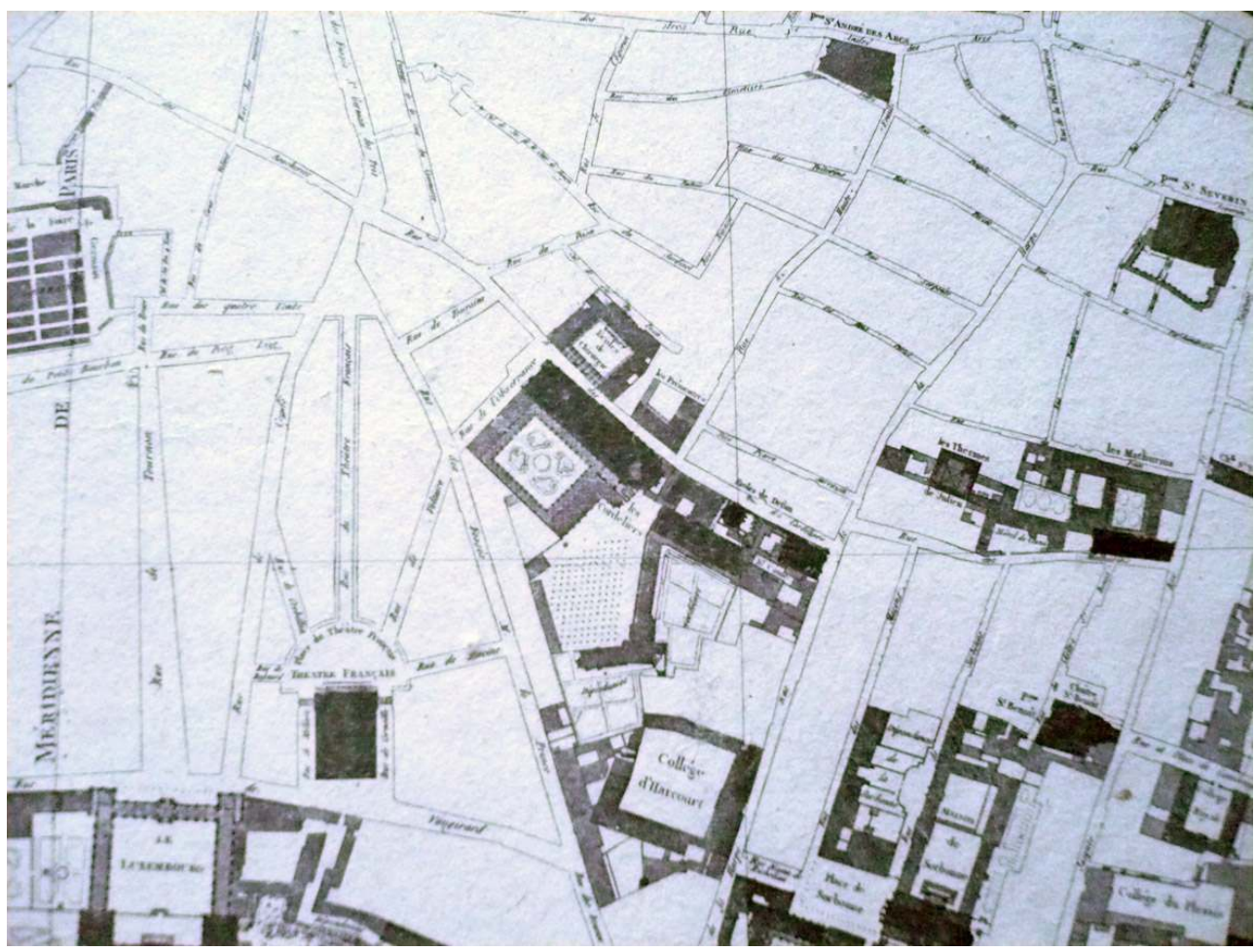

Le quartier de l'Université dans le plan de Verniquet (1791), partie occidentale. Source : Bibliothèque nationale de France, cartes et plans.

(c) BnF. 
Figure 25

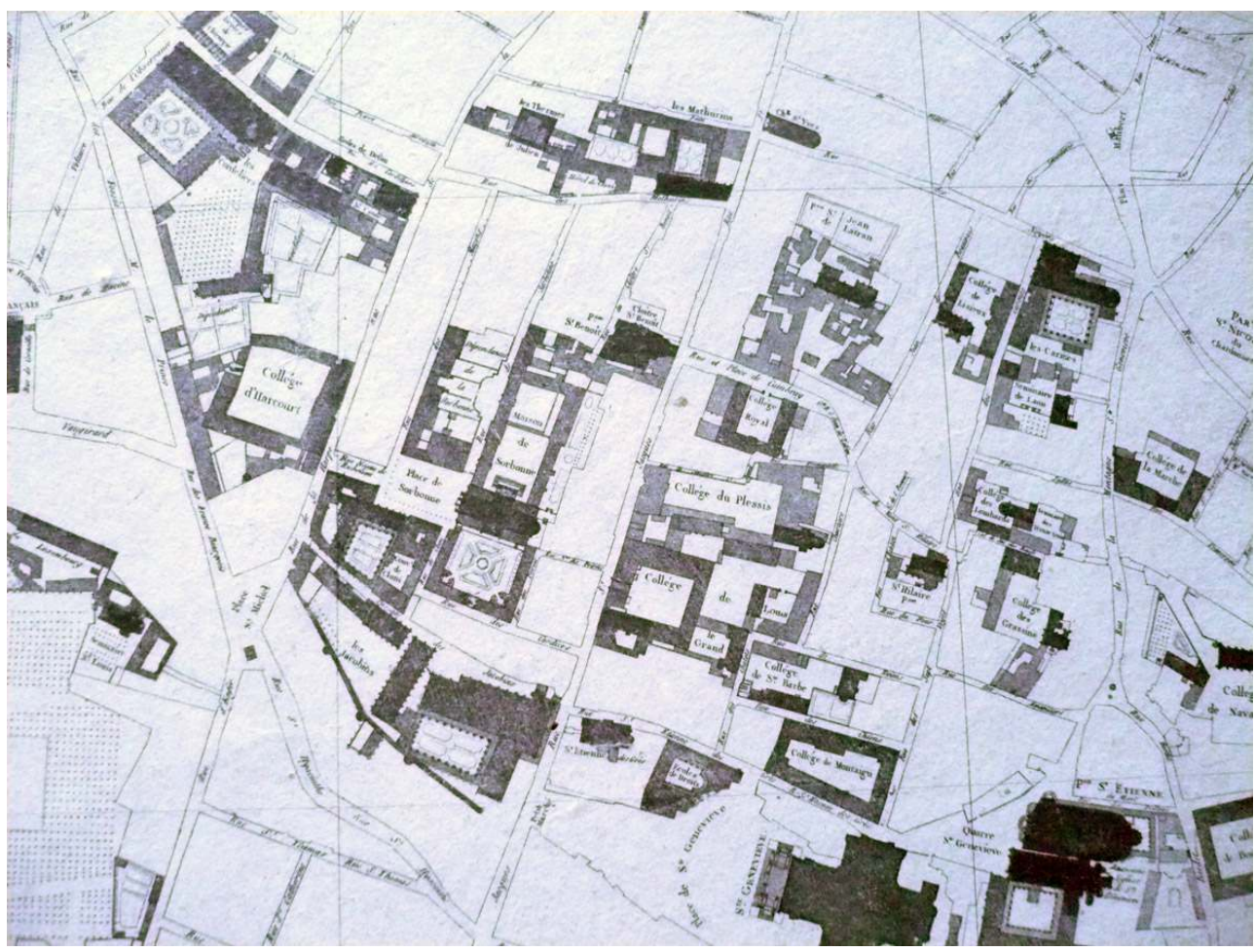

Le quartier de l'Université dans le plan de Verniquet (1791), partie centrale. Source : Bibliothèque nationale de France, cartes et plans.

(C) BnF. 


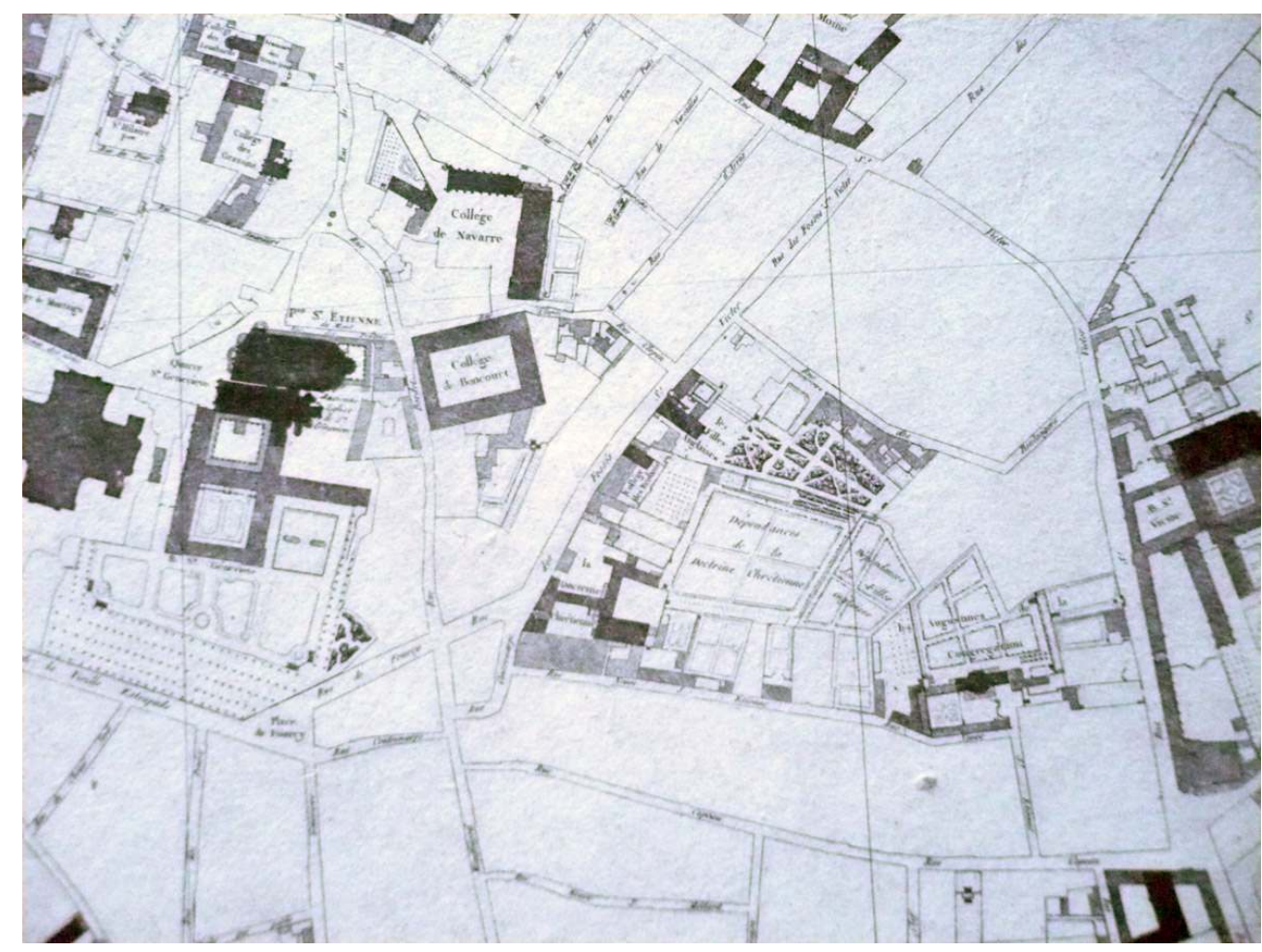

Le quartier de l'Université dans le plan de Verniquet (1791), partie orientale. Source : Bibliothèque nationale de France, cartes et plans.

(C) BnF.

Publié en 1795, le plan de Verniquet dépeint une ville qui n'existe déjà plus, puisque des pans entiers de celle-ci, administrations, hôtels particuliers, hôpitaux, églises, couvents et institutions scolaires, changent de main à la faveur de cette gigantesque partie de redistribution des cartes que constitue la vente des biens nationaux. Cela est particulièrement vrai pour le quartier des écoles et pour son prolongement méridional, compris entre le sommet de la Montagne et le Val de Grâce.

Paris a été le théâtre aux $\mathrm{XVII}^{\mathrm{e}}$ et $\mathrm{XVIII}{ }^{\mathrm{e}}$ siècles de multiples embellissements tant urbanistiques qu'architecturaux. Cependant, alors que la rive droite et l'ouest de la rive gauche (profondément remodelé par le lotissement du faubourg Saint-Germain et les perspectives des Invalides ou de l'École Militaire) ont été bouleversés dans leur physionomie, l'est de la rive gauche n'a guère connu que la construction de nouveaux édifices, et les projets urbains y ont été de médiocre ampleur. Le lotissement du quartier de l'Odéon (fig. 27) et l'aménagement des abords de la nouvelle église Sainte-Geneviève (fig. 28) font bien entendu exception, mais le premier est d'assez petite taille et situé à la marge extérieure du quartier universitaire, tandis que le second est encore en cours : sur le plan de Verniquet des pointillés indiquent l'emplacement prévu pour un bâtiment symétrique à celui de l'École de Droit ${ }^{60}$ et la perspective de la rue percée dans l'axe du portail occidental de l'église bute rapidement sur la rue Saint-Jacques. En revanche, les marges occidentales et orientales du quartier ont vu l'installation de très vastes domaines, appelés à perdurer au-delà de la Révolution et dont l'emprise territoriale va durablement limiter le champ de possibles de la géographie universitaire. Il s'agit à l'ouest, du Palais et Jardin du Luxembourg, adossé aux terrains des Chartreux, et, à l'est, 
du Jardin du roi, futur Muséum d'Histoire Naturelle. Non loin de ce dernier, à l'intersection du quai Saint-Bernard et de la rue des fossés Saint-Bernard, est déjà localisée la Halle aux Vins ${ }^{61}$, promise à une extension considérable sous l'Empire (14 ha) et qui sera pendant près de cent ans l'Eldorado inaccessible des universitaires, scientifiques ou médecins.

Figure 27

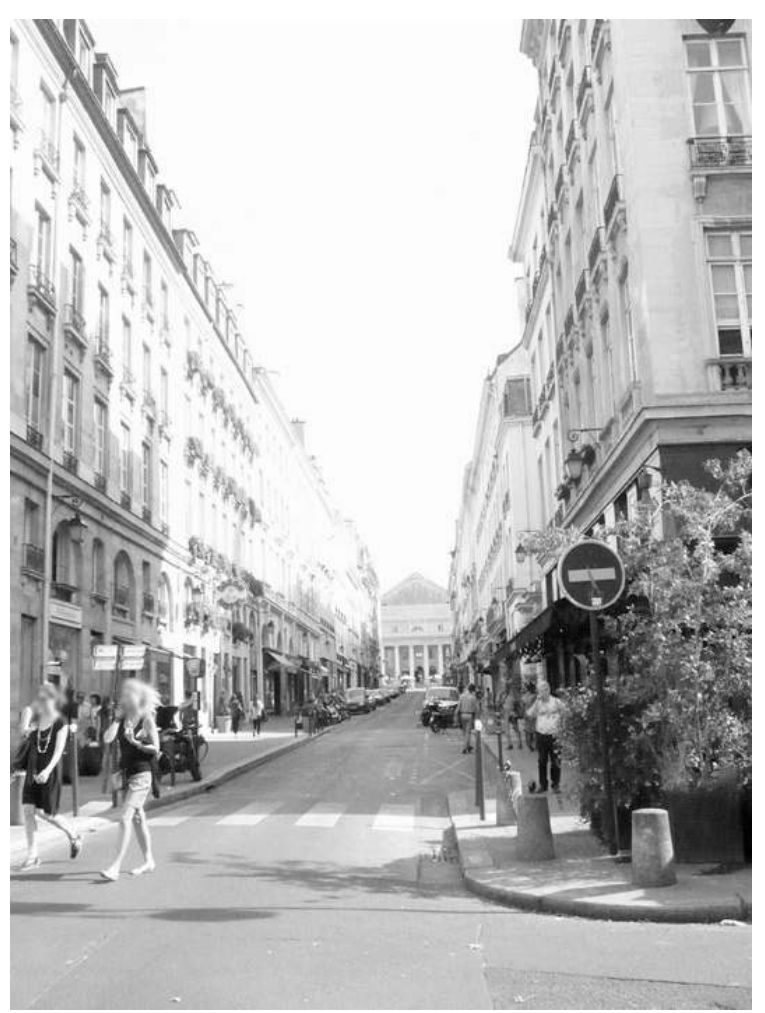

La rue de l'Odéon.

PHOT. HOTTIN, CHRISTIAN, 2014. (C) HOTTIN, CHRISTIAN. 


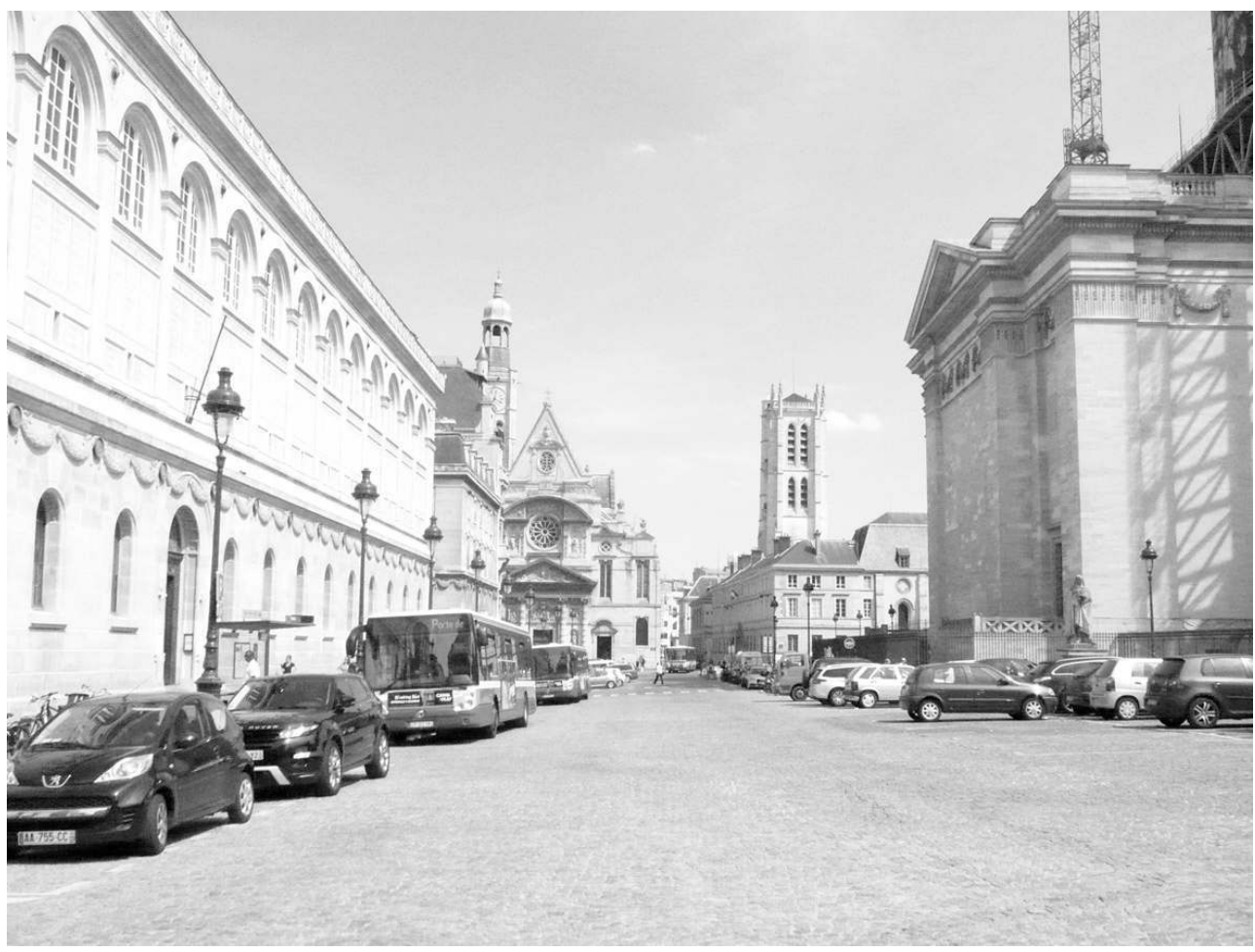

La place du Panthéon.

PHOT. HOTTIN, CHRISTIAN, 2014. (C) HOTTIN, CHRISTIAN. dont le tracé est visible en plan et dans la toponymie : rues des fossés Saint-Germain-desPrés, Monsieur le Prince, Saint-Victor et Saint-Bernard), on pourrait croire que rien ou presque n'a changé depuis la fin du Moyen Âge. Les grands couvents (Cordeliers, Jacobins, etc.) et les nombreuses églises ou paroisses (Saint Côme, Saint-Étienne, Saint-Nicolas, Saint-Yves, etc.) sont toujours là, tout comme les collèges. À y regarder de plus près, ceux-ci occupent pourtant un périmètre beaucoup plus restreint qu'au $\mathrm{XV}^{\mathrm{e}}$ siècle : tous ceux qui se trouvaient dans la partie basse du quartier ont disparu, à l'exception du Collège du Cardinal Lemoine ${ }^{62}$. Les dix collèges de plein exercice, les seuls qui subsistent alors, ainsi que les établissements d'enseignement extérieurs à l'Université, sont massés à l'intérieur d'une ellipse orientée nord-ouest - sud-est et positionnée sur le flanc de la Montagne Sainte-Geneviève. Par exception, quelques fondations plus récentes (Collège des Irlandais - non indiqué sur le plan - et collège des Écossais) sont situées en bordure des rues reprenant le contour de la vieille enceinte ${ }^{63}$.

La topographie d'ensemble du quartier demeure inchangée: les voies les plus importantes ont une orientation nord-est - sud-ouest, et de nombreuses rues de moindre gabarit (Sorbonne, Jean de Beauvais, des Carmes, des Bernardins) sont parallèles à la rue Saint-Jacques. Les voies orientées dans le sens ouest-est sont beaucoup moins nombreuses, de faible largeur et de médiocre longueur, en dehors de la section formée par la rue des noyers prolongée par la rue Saint-Victor. Cette faiblesse des liaisons parallèles au fleuve va elle aussi perdurer, en dépit de nombreux projets, jusqu'au percement de la rue des Écoles et du boulevard Saint-Germain. 
Figure 29

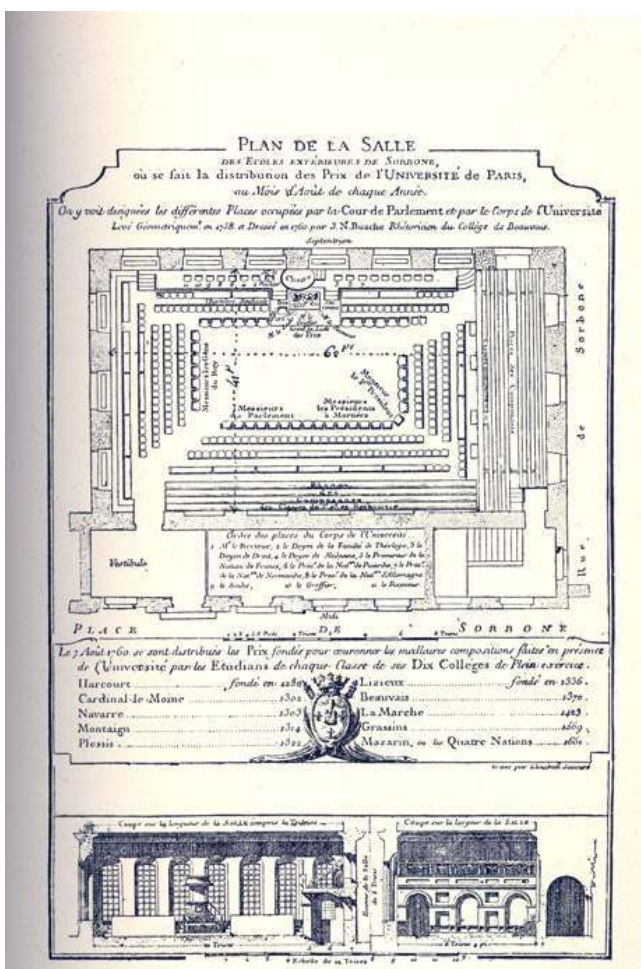

Les écoles extérieures de la Sorbonne, détruites au XIXe siècle. Source : GREARD, Octave. Nos adieux à la vieille Sorbonne. Paris : Hachette, 1893, p. 215. 
Figure 30

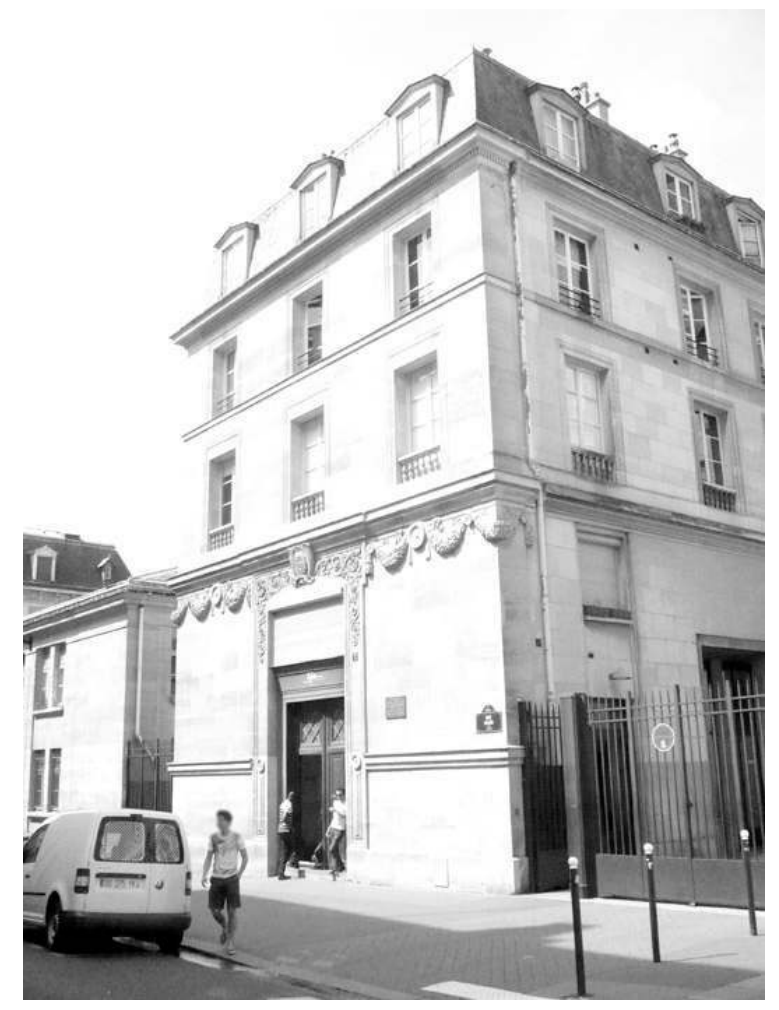

L'ancienne entrée du collège Sainte-Barbe, élevée par Th. Labrouste au XIXe siècle, actuellement entrée de la bibliothèque Cujas.

Phot. Hottin, Christian, 2014. (c) Hottin, Christian. 
Figure 31

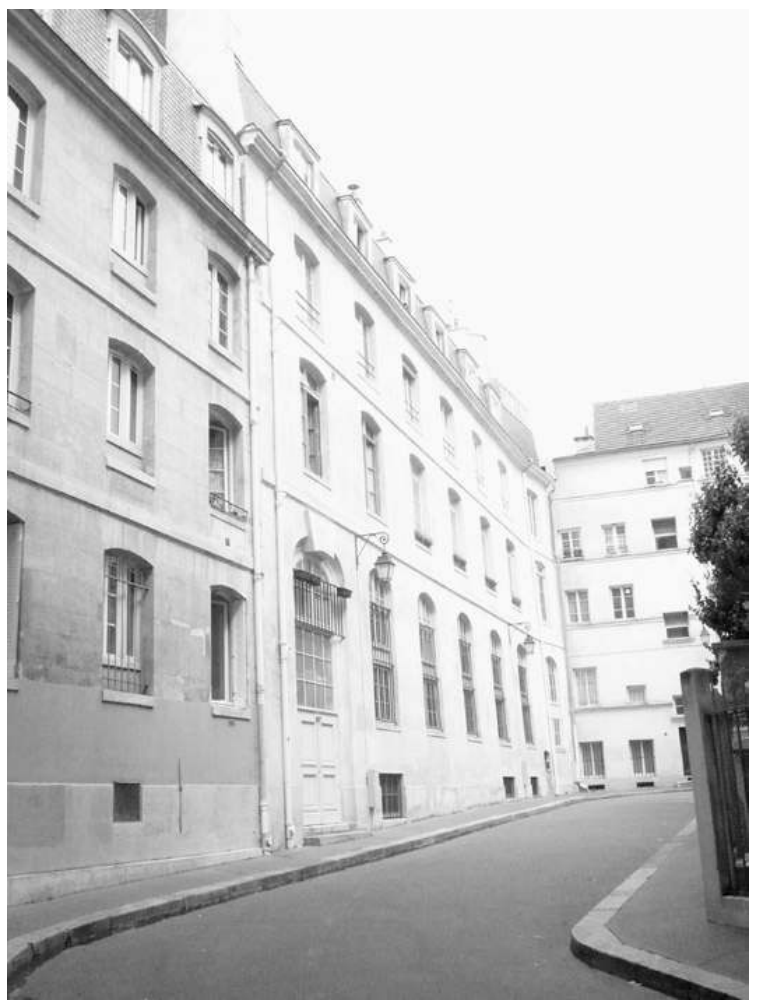

Le collège des Lombards, actuellement maison particulière.

Phot. Hottin, Christian, 2014. (c) Hottin, Christian.

Quant aux établissements, ils se répartissent toujours en plusieurs groupes. À l'ouest de la rue de la Harpe se trouvent l'École de Chirurgie (sur le site du Collège de Bourgogne), l'École gratuite de Dessin et le Collège d'Harcourt (fig. 29). Entre la rue de la Harpe et la rue Saint-Jacques, c'est la "Maison de Sorbonne ", reconstruite par Lemercier sous Richelieu et complétée au sud par la cour du Collège de Calvi. Ses dépendances, dont la salle du concours général ${ }^{64}$, sont comprises entre le côté nord de la place de Sorbonne, la rue du même nom et celle des Maçons (actuelle rue Champollion). Le groupe central, le plus important, est implanté entre les rues Saint-Jacques et de la Montagne SainteGeneviève. S'y retrouvent des collèges anciens (du Plessis, Sainte-Barbe (fig. 30), Montaigu, de Lisieux ou des Lombards) (fig. 31) ou plus récents (Louis-le-Grand, des Grassins), ainsi que les bâtiments neufs de certaines institutions d'enseignement (Faculté de Droit et Collège Royal). L'ancien collège Louis-le-Grand est en outre, comme on l'a vu, le siège de l'Université et de sa bibliothèque. Enfin, le groupe oriental, à l'est de la Montagne, comporte le collège de la Marche (créé en 1362 au nord de la place Maubert, dans les locaux du défunt collège de Constantinople, mais implanté dès 1420 rue SainteGeneviève ${ }^{65}$ ), le séminaire des Trente-trois (fig. 32), le vaste Collège de Navarre, uni à celui de Boncourt en pleine reconstruction (fig. 33), celui du Cardinal Lemoine et celui des Écossais. 
Figure 32

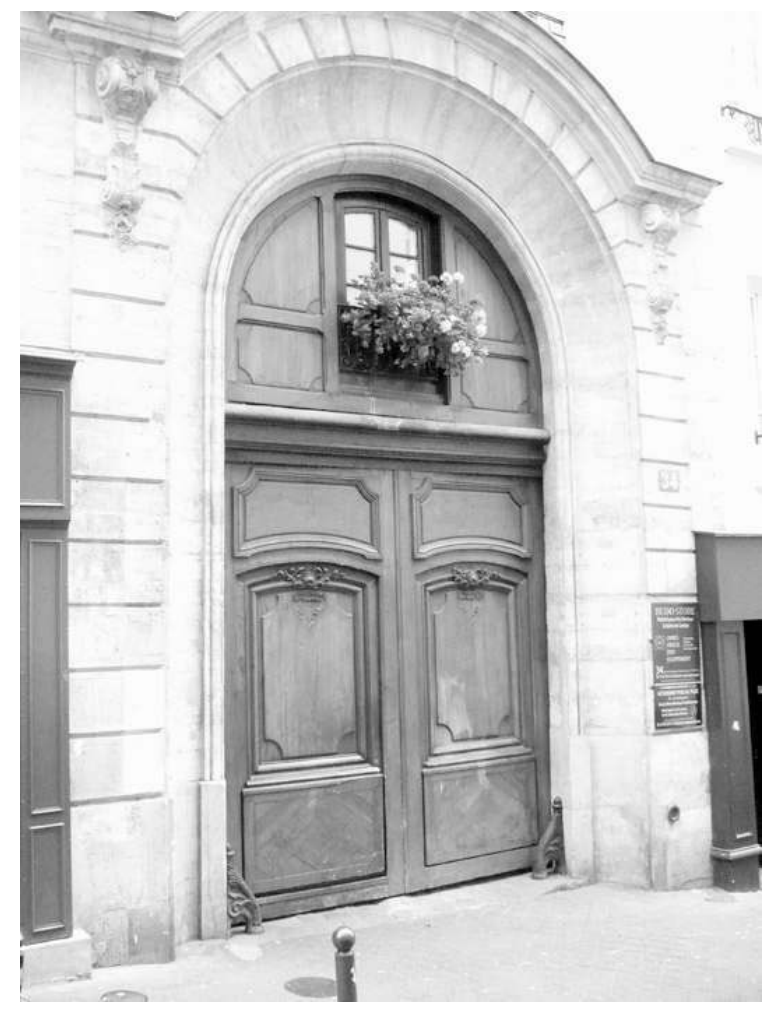

Le Séminaire des Trente-trois, actuellement maison particulière. Phot. Hottin, Christian, 2014. (c) Hottin, Christian. 
Figure 33

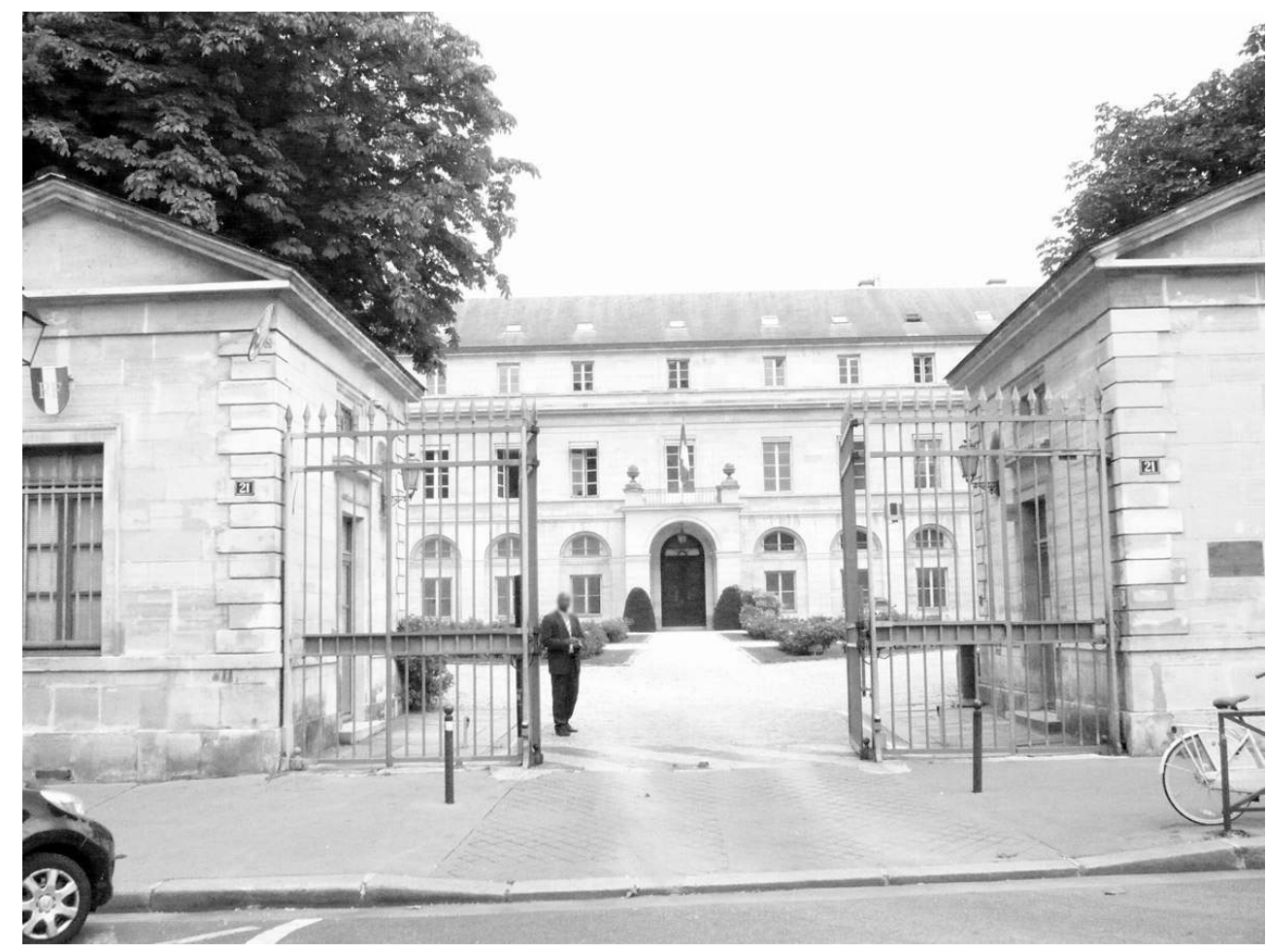

Le Collège de Boncourt, achevé au XIXe siècle, actuellement ministère de l'Enseignement supérieur et de la recherche.

Phot. Hottin, Christian, 2014. (c) Hottin, Christian.

Beaucoup moins nombreux que par le passé, les établissements sont en revanche sensiblement plus vastes. Les plus importants - la Sorbonne, le Collège d'Harcourt, celui de Montaigu ou celui de Louis-le-Grand - s'organisent autour d'une cour centrale plus ou moins régulière, éventuellement complétée par d'autres réservées aux dépendances. L'importance du patrimoine foncier de quelques collèges est manifeste. Celui de Navarre ne comporte qu'une étroite entrée sur la rue de la Montagne Sainte-Geneviève, mais, après un long passage coudé, elle débouche sur une vaste cour intérieure fermée au nord par la chapelle, au sud par la bibliothèque et à l'est par le récent bâtiment des bacheliers ${ }^{66}$ . Ce dernier donne, à l'arrière, sur un petit jardin. Le Collège du Cardinal Lemoine, pour sa part, comporte une vaste cour et ses jardins s'étendent presque jusqu'au quai de la Tournelle.

En résumé, la physionomie du quartier des écoles vers 1789 , quand on la compare à celle observée à la fin de l'époque médiévale, paraît avoir subi une double transformation: le périmètre de l'espace universitaire s'est considérablement réduit, mais cette contraction générale, qui s'accompagne de la disparition de nombreux établissements, va de pair avec la relative dilatation de celles des institutions qui ont survécu: si elles n'occupent pas toujours des superficies plus importantes, elles comptent souvent de plus nombreux bâtiments.

Enfin, il faut dire un mot des terrains qui s'étendent en pente douce du sommet de la Montagne Sainte-Geneviève jusqu'au Val de Grâce ${ }^{67}$. À la fin du XVIII ${ }^{e}$ siècle, ce vaste secteur compris entre la rue Saint-Jacques et la rue Mouffetard est encore peu peuplé, peu construit et traversé de part en part par une seule voie de quelque importance, la rue 
des Postes. En revanche, il est le siège d'un très grand nombre de maisons religieuses, souvent féminines, qui possèdent sur l'arrière de leurs bâtiments conventuels de vastes jardins. Sont ainsi présentes sur la rue Saint-Jacques, du nord au sud: les sœurs de la Visitation Sainte-Marie, les Ursulines, les Feuillantines, les Bénédictines anglaises et l'abbaye du Val de Grâce. Le long de la rue des Postes, on trouve successivement: les Eudistes, le Séminaire anglais, celui du Saint-Esprit (fig. 34), qui fait face au couvent de Sainte-Aure, puis les Orphelines, le couvent de la Présentation, celui de Saint-Michel et celui de la Providence, ainsi que leurs dépendances. Autant les importants domaines de l'ouest et de l'est ne vont cesser de limiter les possibilités d'extension du quartier des écoles, autant ceux du sud vont constituer tout au long du XIX ${ }^{\mathrm{e}}$ et jusqu'au XX $\mathrm{XX}^{\mathrm{e}}$ siècle, au fur et à mesure de leur démantèlement, une réserve foncière appréciable pour les nouvelles institutions d'enseignement supérieur ou les annexes créées par les plus anciennes d'entre elles.

Figure 34

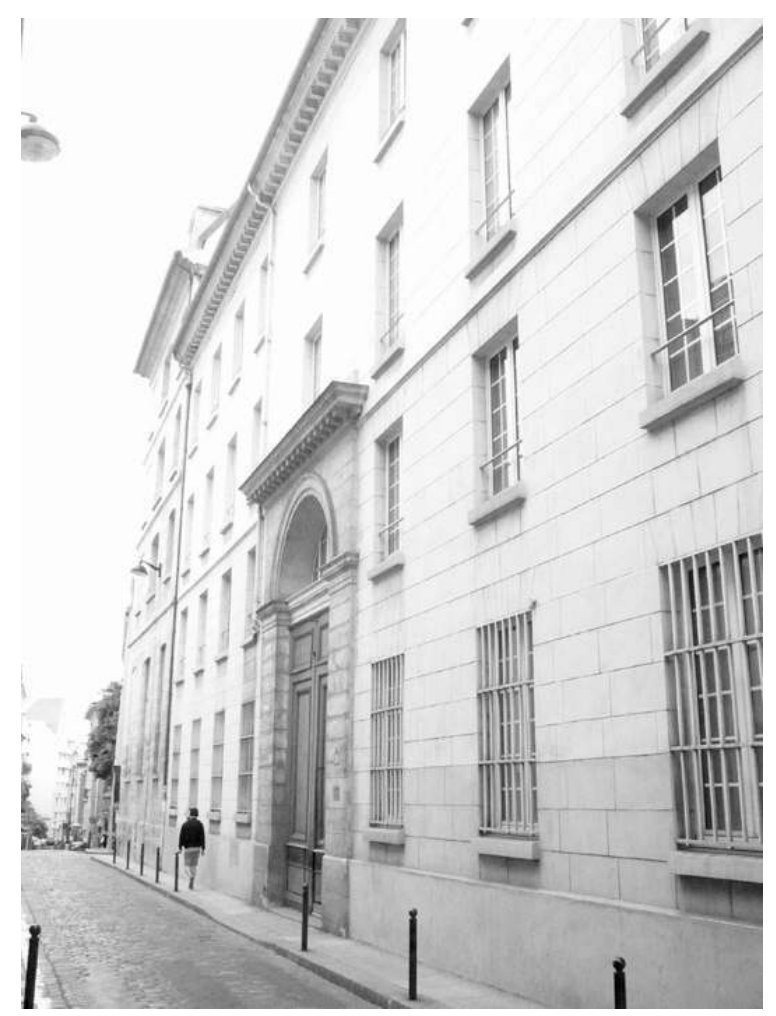

Le séminaire du Saint-Esprit.

PHOT. HOTTIN, CHRISTIAN, 2014. (C) HOTTIN, CHRISTIAN.

Cependant, le principal fait nouveau, quoique invisible sur le plan de Verniquet, réside dans l'existence d'écoles spéciales, totalement étrangères à l'Université et implantées délibérément hors de son quartier historique. La plus ancienne, celle des Ponts et Chaussées, a déjà déménagé plusieurs fois : installée de 1747 à 1750 dans la rue SaintAvoye, au coin de la rue Simon-le-Franc, elle loge de 1751 à 1756 dans une maison de la rue des Blancs-Manteaux. Elle ne reste ensuite que quatre ans dans l'ancien Hôtel de Guénégaud, à l'angle de la rue des Quatre-Fils (fig. 35) et en 1771 un nouveau transfert la conduit au coin de la rue de la Perle et de la rue de Thorigny, dans l'ancien Hôtel Libéral Bruant (fig. 36). 
Figure 35

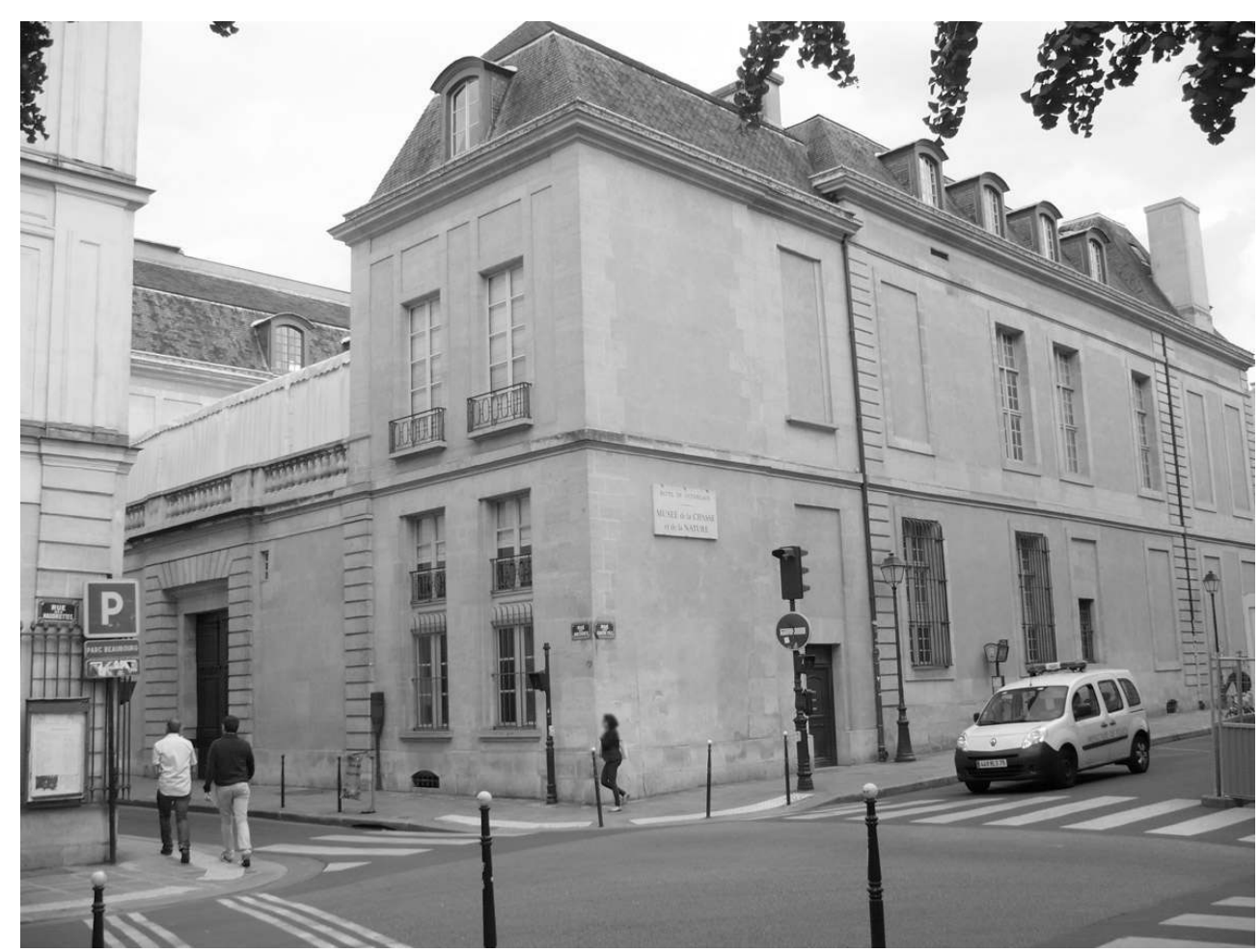

L'hôtel de Guénégaud, actuellement musée de la Chasse.

Phot. Hottin, Christian, 2014. (c) Hottin, Christian. 
Figure 36

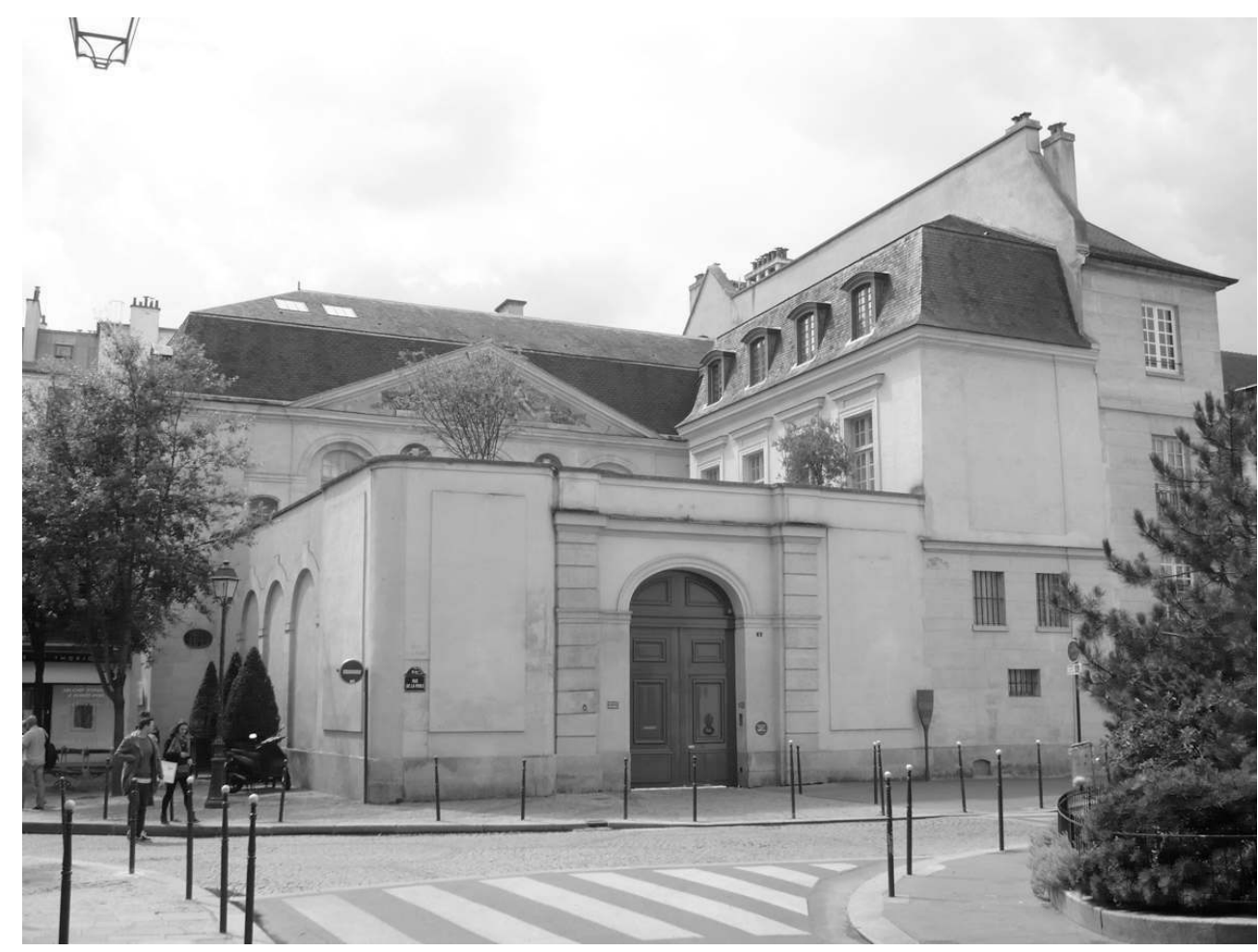

L'hôtel Libéral Bruant, anciennement musée de la serrurerie, actuellement galerie d'art.

Phot. Hottin, Christian, 2014. (c) Hottin, Christian.

Le succès de l'établissement incite Perronet à chercher en 1786 un nouveau bâtiment, et il arrête son choix sur un hôtel de la rue Saint-Lazare, au coin de la rue des Trois-Frères. Telle sera la demeure de l'école jusqu'en $1796^{68}$. Toutes ces implantations ont en commun d'être parfaitement étrangères au quartier et à l'architecture universitaire traditionnels : situées sur la rive droite, souvent dans le Marais, elles consistent principalement en appropriation d'hôtels particuliers. Plus récente et plus petite, l'École des Mines, établie en 1784, repose presque entièrement sur la personnalité de son fondateur, Balthasar Georges Sage, et sur ses collections : elle est sans peine logée dans un salon du nouvel Hôtel des Monnaies ${ }^{69}$. Enfin, l'École Vétérinaire de Paris, premier rejeton de celle créée à Lyon par Bourgelat, trouve aussi à se loger hors du quartier Latin. En 1764 Bourgelat quitte Lyon, laissant à l'abbé Rozier la direction de son établissement hippiatrique. Il se rend à Paris et installe sommairement une première école rue Sainte-Appoline, près des boulevards. Elle est fréquentée par des élèves venus de Lyon ${ }^{70}$. Très simple, l'édifice consiste en un pavillon doté d'un grand salon et de deux pièces au rez-de-chaussée, complété par deux petites pièces à l'étage. Le 27 décembre 1765, Bourgelat rachète au baron de Bormes, en grandes difficultés financières, son château d'Alfort: il vient de trouver le domaine idéal pour son projet scientifique et éducatif, à la fois proche de Paris et doté d'une superficie suffisante pour le développement futur de son établissement. L'installation des élèves a lieu pendant l'été $1766^{71}$. Il semblerait donc que, pour les fondateurs d'établissements totalement étrangers à l'esprit universitaire, la recherche d'une implantation durable ne passe pas par le quartier des écoles. 


\section{Conclusion : Quelle spécificité parisienne?}

47 émigrer, sur la rive gauche de la Seine. Divers hasards et l'heureux voisinage de deux abbayes accueillantes aux idées nouvelles ont été à l'origine d'une formidable concentration d'activité intellectuelle, rayonnante au point qu'en 1231, «Paris, Mère des sciences, brille chère à nos cœurs, comme une seconde Cariath Sapher, la cité des lettres " ${ }^{72}$. Un siècle plus tard est forgée l'idée que cet incroyable succès n'est pas le fruit du hasard, mais qu'il s'inscrit dans une logique, celle de la translatio studii, selon laquelle le studium, la forme la plus élevée de l'enseignement, se déplace au fil des siècles en s'implantant au sein des civilisations les plus brillantes: parti d'Égypte, il est passé à Athènes, puis de là à Rome, et enfin à Paris, où il aurait été amené par Charlemagne ${ }^{73}$. Voici reposée la question de la relation entre l'esprit, par essence immatériel, de l'institution universitaire et sa nécessaire inscription dans l'espace.

L'idée volontiers avancée d'une université médiévale presque entièrement immatérielle, si elle ne manque pas de validité pour ce qui concerne ses subdivisions que sont les nations ou les facultés, est à nuancer en raison de l'existence d'un maillage dense de lieux bâtis qui font l'université et dans lesquels la dimension universitaire vient comme en surimpression de fonction principale, qui est religieuse, les deux étant du reste indissolublement liées sous l'Ancien Régime: c'est le cas des églises qui accueillent assemblées et archives, cela vaut également pour les couvents dont la fondation est motivée par l'existence de l'Université et qui abritent étudiants et chaires de théologie. Passé 1250, le développement massif des collèges fait de ceux-ci la manifestation architecturale privilégiée de la vie universitaire. Ce phénomène ne cessera de s'accentuer, puisqu'à l'époque moderne de somptueux collèges sont construits ou reconstruits, alors que les locaux des facultés (droit ou médecine principalement) restent très modestes. Même si elle ne s'incarne pas dans un bâtiment privilégié, l'Université s'inscrit incontestablement dans un espace, celui de la rive gauche, au point de désigner cette dernière par métonymie. Cette trope perd pourtant de sa véracité au fil des siècles : alors que toute la rive gauche, ou presque, était universitaire à la fin $\mathrm{du} \mathrm{XV}^{\mathrm{e}}$ siècle, ce n'est plus le cas en 1789. Un double phénomène s'est entre-temps produit: les faubourgs se sont considérablement développés - même si la rive droite reste la plus peuplée - et le quartier des écoles s'est sensiblement rétracté, pour ne plus couvrir que le flanc nord de la Montagne Sainte-Geneviève. Réduit comme une peau de chagrin, le quartier Latin est à l'image de l'institution qui l'occupe: archaïque et replié sur lui-même. Les projets d'urbanisme, comme les réformes de l'institution, sont timides ou inaboutis. Sa légitimité à incarner la science dans l'espace est contestée par les dirigeants des écoles techniques qui préfèrent pour leur établissement d'autres localisations dans Paris, quand ce n'est pas hors de la ville: à la même époque Bourgelat, Sage ou Perronet défient l'institution universitaire en développant en dehors de ses cadres un enseignement de haut niveau, mais à finalité pratique. 
Figure 37

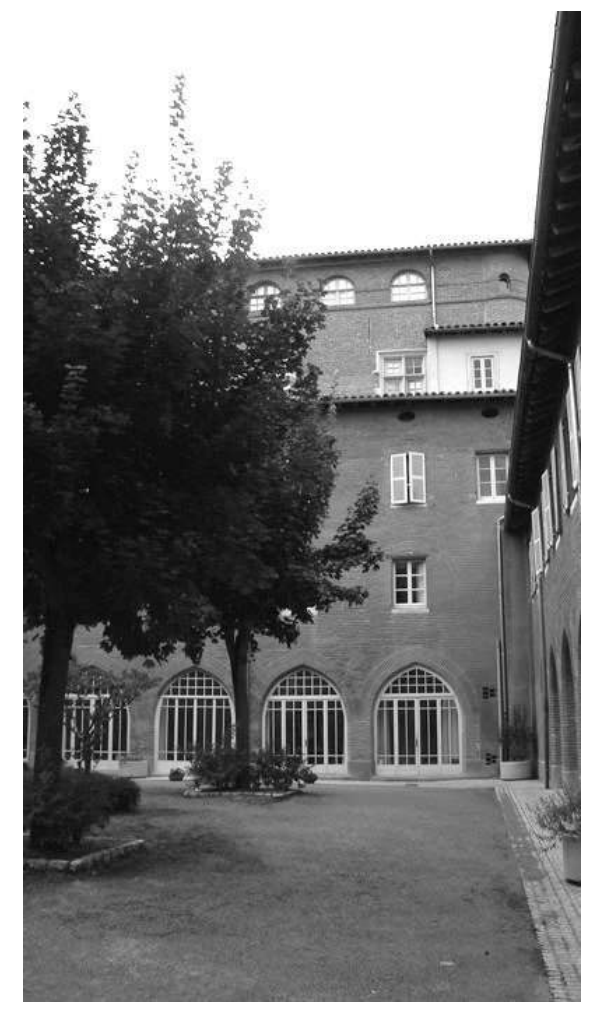

Toulouse, le collège de Foix, actuellement résidence étudiante. Phot. Hottin, Christian, 2014. (c) Hottin, Christian.

Figure 38

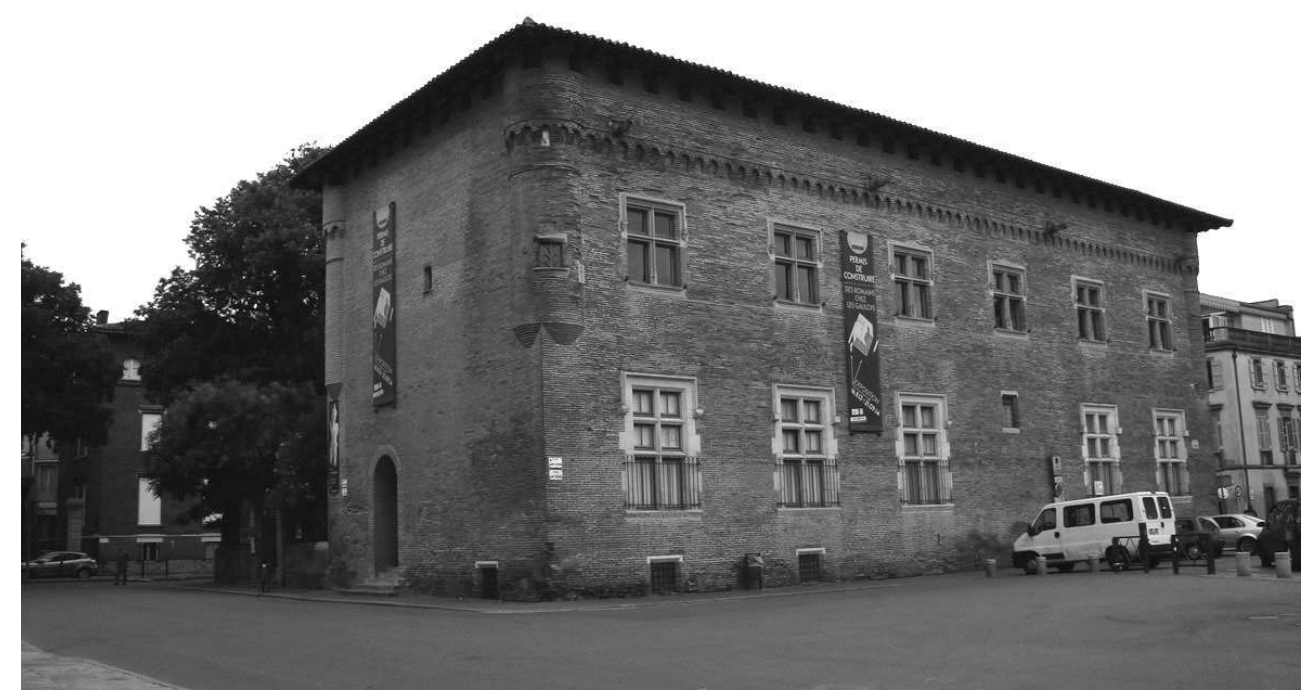

Toulouse, le collège Saint-Raymond, actuellement musée Saint-Raymond. Phot. Hottin, Christian, 2014. (c) Hottin, Christian. 
Figure 39

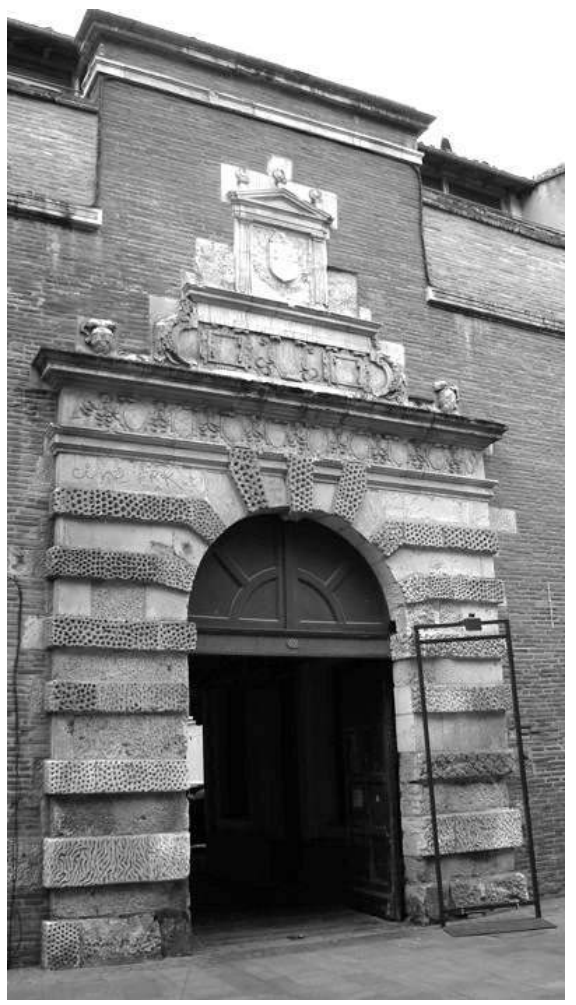

Toulouse, le collège de l'Esquile, actuellement cinémathèque. Phot. Hottin, Christian, 2014. (c) Hottin, Christian.

Figure 40

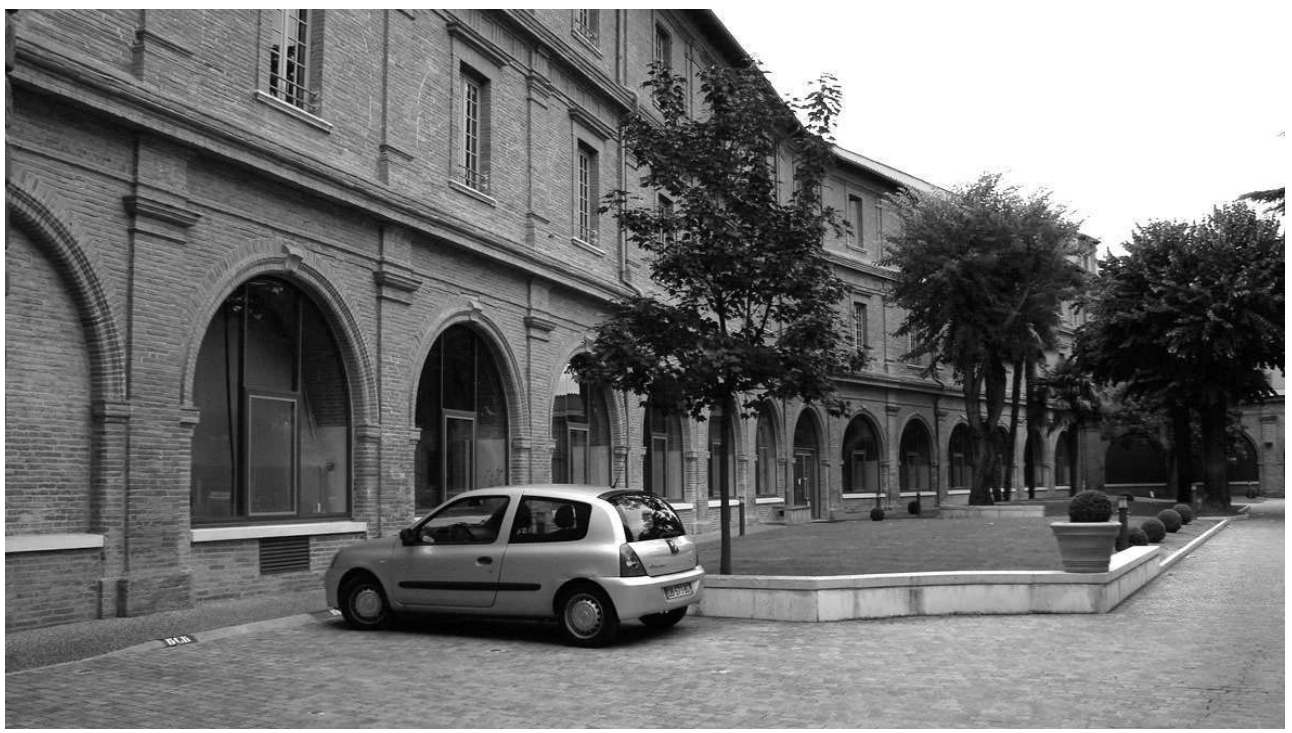

Toulouse, le collège de l'Esquile, actuellement services administratifs. Phot. Hottin, Christian, 2014. (c) Hottin, Christian. 


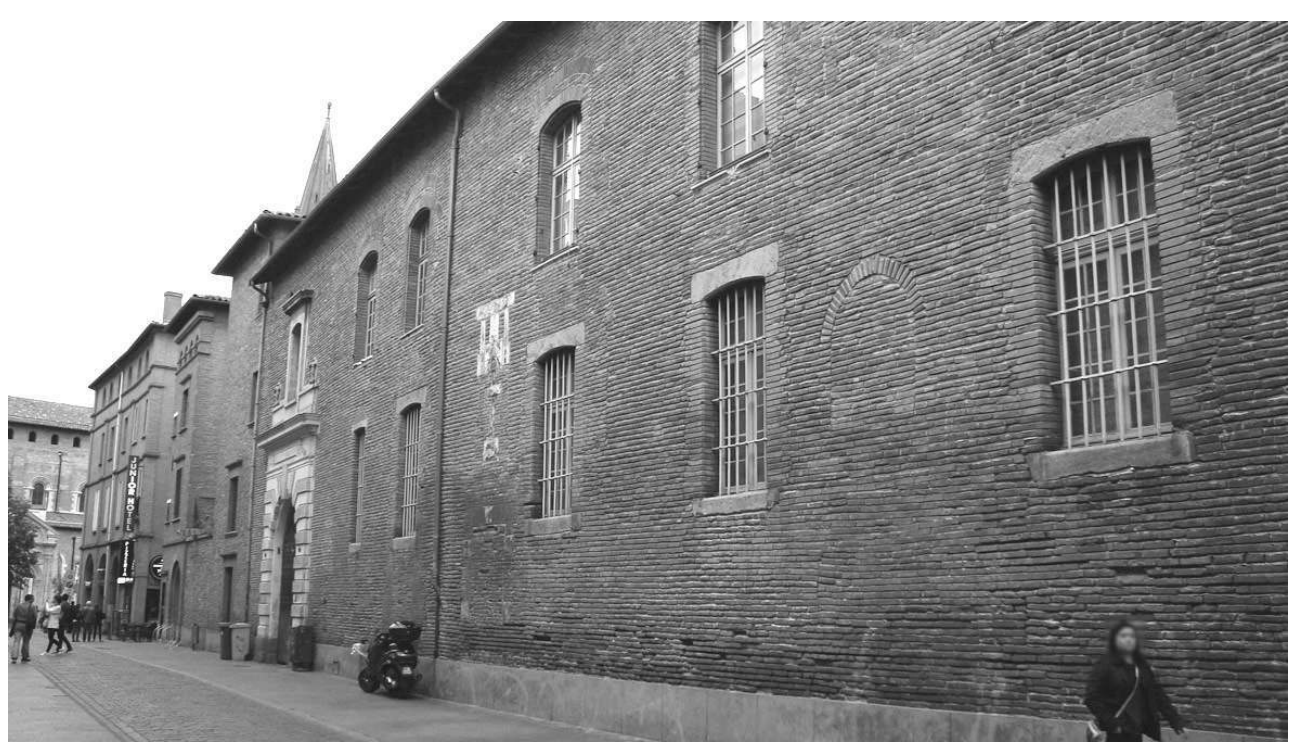

Toulouse, le collège de Périgord, actuellement école supérieure d'audiovisuel.

Phot. Hottin, Christian, 2014. (c) Hottin, Christian.

Sans multiplier les points de comparaison, on peut tenter d'évaluer la spécificité de la géographie historique de l'Université de Paris en évoquant quelques exemples provinciaux ou étrangers. Comme celle de Paris, l'Université de Toulouse a prospéré en limite de la ville traditionnelle, dans le bourg de l'abbaye Saint-Sernin : ses principaux collèges (de Foix, de l'Esquille, de Périgord, Saint-Raymond) (fig. 37, 38, 39, 40, 41) et voisinent avec les couvents des ordres mendiants, Cordeliers ou Jacobins. Au XVI ${ }^{e}$ siècle, les jésuites s'établissent à proximité immédiate de ces derniers. Les ressemblances sont donc nombreuses. Tout au contraire, les Universités anglaises d'Oxford et Cambridge, nées comme Paris et Toulouse au XIII ${ }^{\mathrm{e}}$ siècle, présentent une morphologie toute différente : à Cambridge, dès le Moyen Âge, l'Université est le corps principal de la ville, les collèges occupent le cœur de l'espace urbain et les plus importants d'entre eux s'alignent le long de la rue centrale. Ces derniers disposent de vastes terrains vierges de toute construction entre la rue et la rivière $\mathrm{Cam}$, et qui constituent dès cette époque une précieuse réserve foncière pour les développements futurs ${ }^{74}$. Comme à Paris, en revanche, les collèges sont l'élément qui structure visuellement le paysage de l'Université : celle-ci est un agrégat de collèges et ils sont le cœur de la vie universitaire. Les bâtiments relevant uniquement de l'université sont peu nombreux. La comparaison s'arrête là : contrairement à ceux de Paris, les collèges anglais sont très richement dotés et ont su faire fructifier leur patrimoine initial : à la fin du XVIII ${ }^{e}$ siècle, ils offrent un visage de prospérité qu'on chercherait vainement sur les bords de la Seine ${ }^{75}($ fig. 42, 43). 


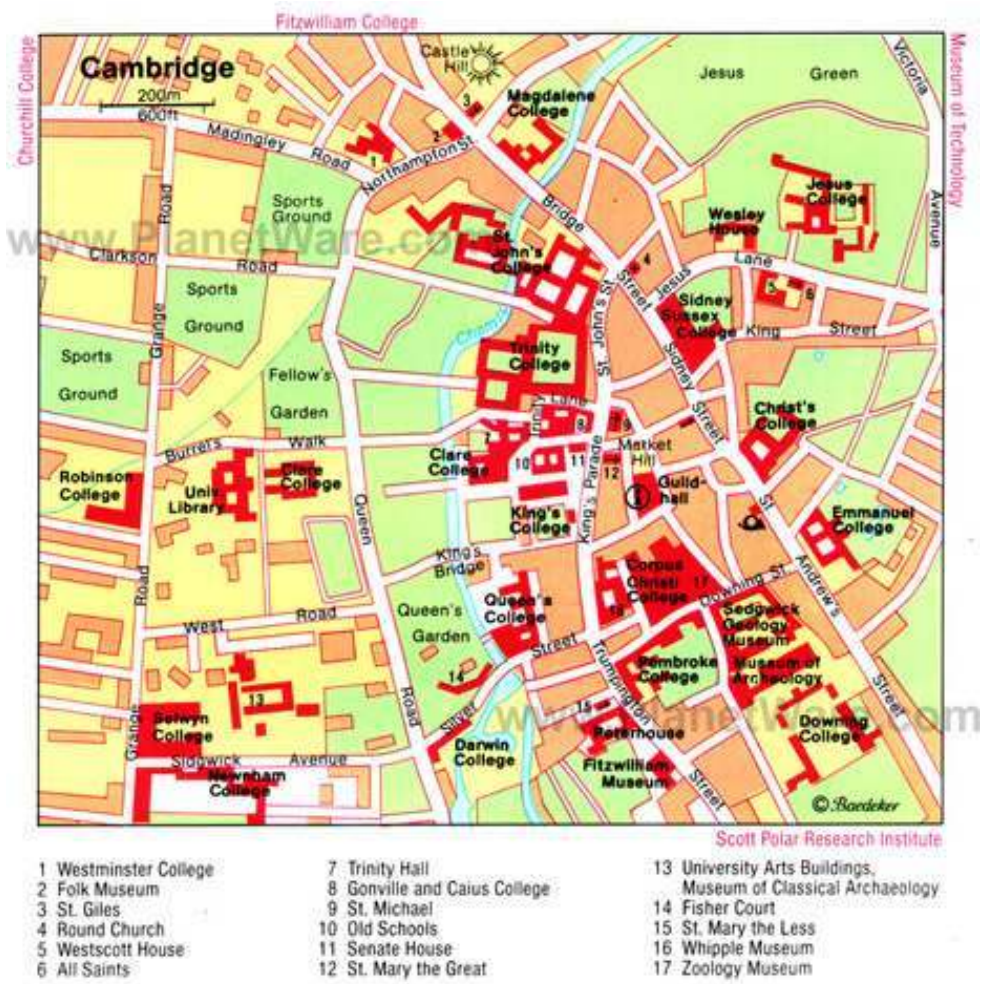

Cambridge, plan de la ville (2014). Scott Polar research Institute (http://www.planetware.com/touristattractions-/cambridge-eng-cmb-cambrig.htm).

Figure 43

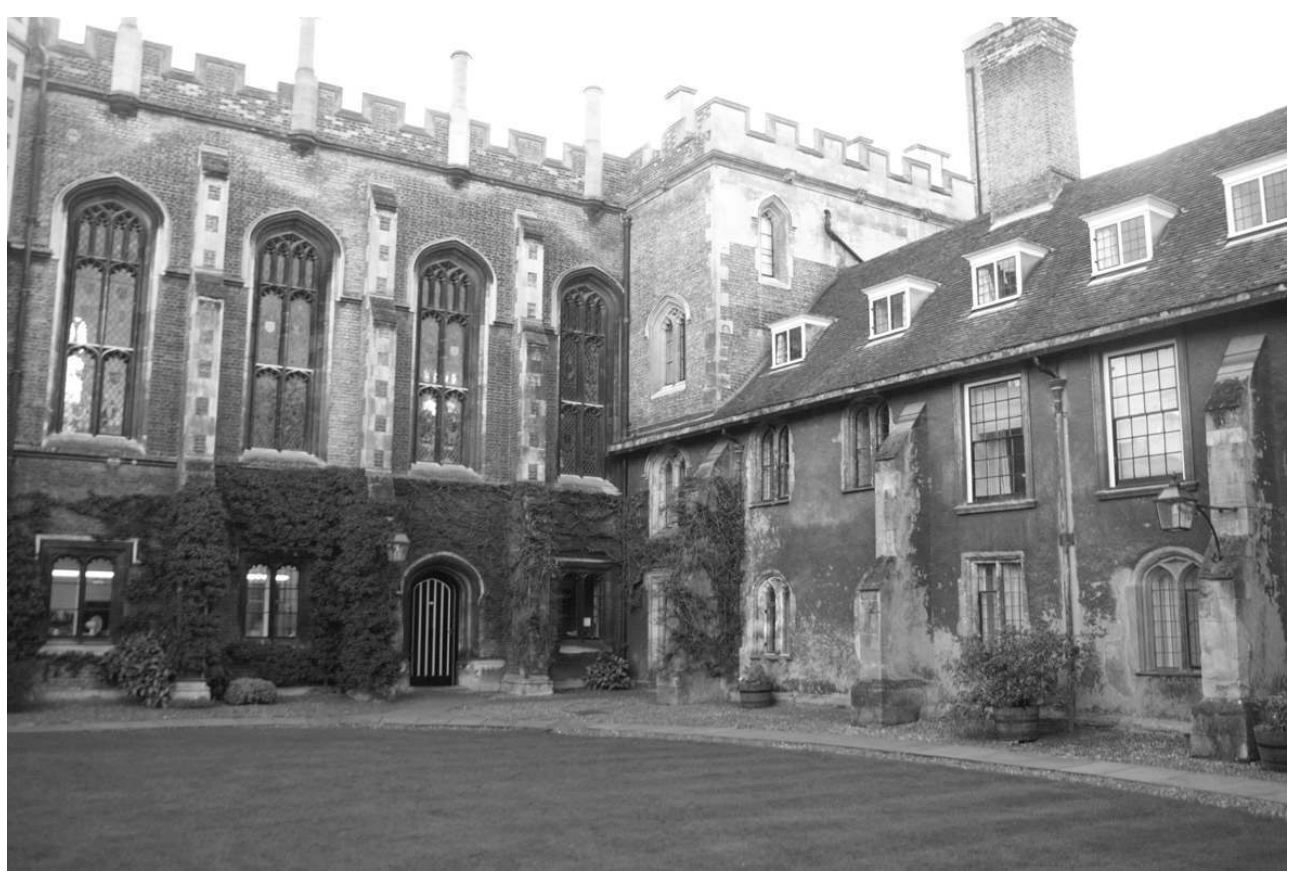

CAMBridge (ANgLETERRE), OLd COURT DE CORPUS CHRISTI COLLEgE.

PHOT. BORTOLOTTO, CHIARA, 2014. () BORTOLOTTO, CHIARA. 
L'Université de Paris se présente donc comme une institution qui est l'une des fonctions, mais non la seule de la ville qui l'accueille. Cette institution s'incarne dans plusieurs séries de bâtiments, collèges ou écoles, dont il faut maintenant tenter de décrire la physionomie à la veille de la Révolution.

\section{NOTES}

*. Ce texte s'inscrit dans le prolongement de recherches précédemment publiées dans In situ, avec lesquelles il forme un triptyque. Voir : HOTTIN, Christian. «Retour sur un patrimoine parisien méconnu : les espaces de transmission du savoir à l'époque moderne (I). De la maison à l'amphithéâtre ». In Situ, revue des patrimoines [en ligne], 2009, n¹0 [consulté le 04/08/2011]. http://www.insitu.culture.fr/article.xsp?numero=10\&id_article=hottin-1343; et HOTTIN, Christian. « Retour sur un patrimoine parisien méconnu : les espaces de transmission du savoir à l'époque moderne (II)* Naissance d'une architecture : quatre projets exceptionnels (ca. 1760 - ca. 1790) ", In Situ, revue des patrimoines [en ligne], 2011, n¹7, [mis en ligne le 18 janvier 2012, consulté le 24 janvier 2013]. URL : http://insitu.revues.org/1069 ; DOI : 10.4000/insitu.1069

1. - Cariath Sepher : Ville royale de Palestine, dans la tribu de Juda, selon le livre de Josué. Cette ville était l'endroit où l'on enseignait les lettres et les sciences. Son nom signifie «la ville du livre ".

2. - D'IRSAY, Stephen. Histoire des universités françaises et étrangères des origines à nos jours, tome I (Moyen Âge et Renaissance). Paris : Picard, 1933, 372 p., p. 153.

3. - L'idée est également développée par Durkheim : DURKHEIM, Émile. L'Évolution pédagogique en France. Paris : Presses universitaires de France (coll. Quadrige), 1938, 403 p., p. 104-105. Voir le site : http://gallica.bnf.fr/ark:/12148/bpt6k57892738/f121.image.r=emile\%20durkheim.langFR.

4. - ROUX, Simone. La rive gauche des escholiers. Paris : éditions chrétiennes (coll. vivre l'Histoire), 1992, 186 p., p. 14.

5. - ROUX, Simone. La rive gauche des escholiers. Paris : éditions chrétiennes (coll. vivre l'Histoire), 1992, 186 p., p. 9.

6. - Il s'agit de l'abbaye d'Abélard en Champagne.

7. - LE GOFF, Jacques. "Quelle conscience l'université médiévale a-t-elle eu d'elle-même?", Miscellanea mediaevalia, vol. III, Beiträge des Mittelalterlichen Menschen, Berlin, 1964, p. 15-29 (repris dans Pour un autre Moyen Âge, Culture en Occident. Dix-huit essais. Paris : Gallimard (bibliothèque des Histoires), 1977, 422 p., p. 181-197). La perception de cette spécificité est également ressentie par ceux qui sont extérieurs au monde urbain: Philippe de Harvengt, de l'ordre des Prémontrés, explique dans ses lettres à Héroald ou Engelbert sa fascination pour Paris et fait l'éloge de cette ville universitaire. Sur l'identité universitaire médiévale, voir aussi : VERGER, Jacques. « La dicte université de Thoulouse est moult notable et très ancienne. Histoire et conscience de soi chez les universitaires toulousains à la fin du Moyen Age ». Saint-Denis et la royauté, études offertes à Bernard Guenée. Paris : Publications de la Sorbonne, 1999, 814 p., p. 593-606 ; et LUSIGNAN, Serge. "Vérité garde le roi ». La construction d'une identité universitaire en France (XIII ${ }^{e}-X^{e}$ siècle). Paris: Publications de la Sorbonne, 1999, $332 \mathrm{p}$.

8. - VERGER, Jacques. Culture, enseignement et société en occident aux XII et XIII ${ }^{\mathrm{e}}$ siècles. Rennes : Presses universitaires de Rennes, 194 p., p. 35. 
9. - TUILIER, André. Histoire de l'Université de Paris et de la Sorbonne, t. I, Des origines à Richelieu. Paris : nouvelle librairie de France, G.-V. Labat éditeur, 1994, 620 p., p. 35.

10. - VERGER, Jacques. Les universités au Moyen Âge. Paris : Presses universitaires de France, 1973, 226 p., p. 26.

11. - Il en fait état dans une lettre écrite postérieurement au Métalogicon. VERGER, Jacques. Culture, enseignement et société en occident aux XII ${ }^{\mathrm{e}}$ et XIII ${ }^{\mathrm{e}}$ siècles. Rennes : Presses universitaires de Rennes, 194 p., p. 40.

12. - TUILIER, André. Histoire de l'Université de Paris et de la Sorbonne, t. I, Des origines à Richelieu. Paris : nouvelle librairie de France, G.-V. Labat éditeur, 1994, 620 p., p. 55.

13. - VERGER, Jacques. Les Universités au Moyen Âge. Paris : Presses universitaires de France, 1973, 226 p., p. 85.

14. - Bien que présents dès 1219 , ils ne se fixent à cet endroit qu'en 1230. TUILIER, André. Histoire de l'Université de Paris et de la Sorbonne, t. I. Paris : nouvelle librairie de France, G.-V. Labat éditeur, 1994, 620 p., p. 51.

15. - VERGER, Jacques. "L'Université de Paris et ses collèges au temps de Jérôme de Moravie ». Les universités françaises au Moyen Âge. E.J. Brill, Leiden, New-York, Köln, 1995, 255 p., p. 53-67, p. 56, précédemment publié dans: Jérôme de Moravie, un théoricien de la musique dans le milieu intellectuel parisien du XIII siècle (Chr. Meyer, éd.). Paris : éditions Créaphis, 1992, p. 43-64.

16. - Pour une histoire détaillée des collèges parisiens, voir: COMPERE, Marie-Madeleine. Les collèges français (XVI ${ }^{e}-X V I I I^{e}$ siècles). Répertoire. 3. Paris. Paris : INRP, 2002, $472 \mathrm{p}$.

17. - Le Collège des Dix-huit clercs, édifié juste en face du parvis de Notre-Dame, est fondé en 1180. VERGER, Jacques. Les universités au Moyen Âge. Paris : Presses universitaires de France, 1973, 226 p., p. 71.

18. - C'est le cas du Collège Saint-Honoré, près de l'église du même nom. TUILIER, André. Histoire de l'Université de Paris et de la Sorbonne, t. I. Paris : nouvelle librairie de France, G.-V. Labat éditeur, 1994, 620 p., p. 100.

19. - PERRAUT, Aurélie. L'architecture des collèges parisiens au Moyen Age (Préface de Dany Sandron). Paris : Presses de l'Université de Paris Sorbonne, 2009 (coll. Cultures et civilisations médiévales, $\mathrm{n}^{\circ} 46$ ). [en ligne] In Situ, revue des patrimoines, 2011, n¹7, URL http:// insitu.revues.org/970.

20. - À la même époque sont fondés à Oxford les collèges de Merton, Balliol et University, ainsi que celui de Peterhouse à Cambridge. Voir : VERGER, Jacques. Les universités au Moyen Âge. Paris : Presses universitaires de France, 1973, 226 p., p. 71.

21. - VERGER, Jacques. "L'Université de Paris et ses collèges au temps de Jérôme de Moravie ». Les universités françaises au Moyen Âge. E.J. Brill, Leiden, New-York, Köln, 1995, 255 p., p. 53-67, p. 62-63, précédemment publié dans : Jérôme de Moravie, un théoricien de la musique dans le milieu intellectuel parisien du XIII siècle (Chr. Meyer, éd.). Paris : éditions Créaphis, 1992, p. 43-64.

22. - Dès 1254, Robert de Sorbon, Guillaume de Chartres et Guillaume de Mémont achètent des maisons dans le quartier des écoles pour y créer une fondation. TUILIER, André. Histoire de l'Université de Paris et de la Sorbonne, t. I. Paris : nouvelle librairie de France, G.-V. Labat éditeur, 1994, 620 p., p. 115-127.

23. - BOURGIN, G. «Quatre actes concernant les origines du Collège d'Harcourt ». Bulletin de la société d'Histoire de Paris et de l'île-de-France, 31, 1904, p. 98-108.

24. - RABUT, Élisabeth. «Les Cholets, Étude historique et topographique d'un collège parisien ». Paris et Île-de-France, Mémoires, 21, 1970, p. 7-95 et 22, 1971, p. 119-231.

25. - GOROCHOV, Nathalie. Le Collège de Navarre de sa fondation (1305) au début de XV ${ }^{\mathrm{e}}$ siècle (1418). Histoire de l'institution, de sa vie intellectuelle et de son recrutement. Paris : Honoré Champion, 1997, 753 p., p. 131-132. Le projet est décrit p. 143. 
26. - GOROCHOV, Nathalie. Le Collège de Navarre de sa fondation (1305) au début de $\mathrm{XV}^{\mathrm{e}}$ siècle (1418). Histoire de l'institution, de sa vie intellectuelle et de son recrutement. Paris : Honoré Champion, 1997, 753 p., p. 131-132. Le projet est décrit p. 151-154.

27. - C'est le cas par exemple à Montpellier autour de trois églises (Saint-Firmin pour les médecins, Sainte-Eulalie pour les juristes et Sainte-Foy pour les artiens) ou à Bologne dans la partie sud de la ville. Selon Jacques Verger, l'unité de la condition universitaire au XIII ${ }^{\mathrm{e}}$ siècle se manifeste par l'intégration des individus dans un processus de travail en commun effectué au sein d'un même quartier. VERGER, Jacques. Les universités au Moyen Âge. Paris: Presses universitaires de France, 1973, 226 p., p. 72-73.

28. - Cartulaire de l'Université de Paris, t. I, n462, cité par : LUSIGNAN, Serge. Vérité garde le roy. La construction d'une identité universitaire en France $\left(\mathrm{XIII}^{\mathrm{e}}-\mathrm{XV}^{\mathrm{e}}\right.$ siècles). Paris : Publications de la Sorbonne, 1999, 332 p., p. 129.

29. - LUSIGNAN, Serge. Vérité garde le roy. La construction d'une identité universitaire en France (XIII ${ }^{\mathrm{e}}-\mathrm{XV}^{\mathrm{e}}$ siècles). Paris : Publications de la Sorbonne, 1999, 332 p, p. 131-135.

30. - LUSIGNAN, Serge. Vérité garde le roy. La construction d'une identité universitaire en France (XIII $-\mathrm{XV}^{\mathrm{e}}$ siècles). Paris : Publications de la Sorbonne, 1999, 332 p, p. 134.

31. - LUSIGNAN, Serge. Vérité garde le roy. La construction d'une identité universitaire en France (XIII ${ }^{\mathrm{e}}-\mathrm{XV}^{\mathrm{e}}$ siècles). Paris : Publications de la Sorbonne, 1999, 332 p, p. 135.

32. - Voir le plan « Le Quartier latin à Paris vers la fin du XIV e siècle », extrait du plan « Paris vers la fin du XIV ${ }^{e}$ siècle », CNRS - Cartographie thématique, 1975. Reproduction : TUILIER, André. Histoire de l'Université de Paris et de la Sorbonne, t. I. Paris : nouvelle librairie de France, G.-V. Labat éditeur, 1994, 620 p., inséré entre les p. 96 et 97.

33. - Il existe par exemple un inventaire des papiers de la nation d'Allemagne conservés à l'église Saint-Cosme-et-Saint-Damien. JULLIEN DE POMMEROL, Marie-Henriette et MONFRIN, Jacques. «Les archives des universités médiévales. Problèmes de documentation ». Revue française de pédagogie, n²7, avril-juin 1974, p. 4-21. Repris dans Archives et sources pour l'histoire de l'enseignement (Thérèse Charmasson éd.). Paris : Comité des travaux historiques et scientifiques, 2005, 391 p., p. 13-45, p. 18-20.

34. - ROUX, Simone. La rive gauche des escholiers. Paris : éditions chrétiennes (collection vivre l'Histoire), 1992, 186 p., p. 39.

35. - FOURIER, Ch. L'enseignement français de l'Antiquité à la Révolution. Précis d'histoire des institutions scolaires par les textes juridiques. Paris : IPN-SEVPEN, 1964, 2 vol., vol. 1, p. 92.

36. - PERRAUT, Aurélie. L'architecture des collèges parisiens au Moyen Âge (Préface de Dany Sandron). Paris: Presses de l'Université de Paris Sorbonne, 2009, 467 p. (Coll. cultures et civilisations médiévales, $\left.\mathrm{n}^{\circ} 46\right)$, p. 83-84.

37. - PERRAUT, Aurélie. L'architecture des collèges parisiens au Moyen Âge (Préface de Dany Sandron). Paris: Presses de l'Université de Paris Sorbonne, 2009, 467 p. (Coll. cultures et civilisations médiévales, $\left.\mathrm{n}^{\circ} 46\right)$, p. 86-87.

38. - VERGER, Jacques. Les universités au Moyen Âge. Paris : Presses universitaires de France, 1973, 226 p., p. 188.

39. - VERGER, Jacques. Les universités au Moyen Âge. Paris : Presses universitaires de France, 1973, 226 p., p. 179.

40. - Indépendamment des spécificités françaises, le phénomène s'observe dans toute l'Europe, comme en témoignent les bibliothèques de la Divinity school d'Oxford (1445), des écoles de Cambridge (1440-1446) ou de Salamanque (1465-1474). VERGER, Jacques. Les universités au Moyen Âge. Paris : Presses universitaires de France, 1973, 226 p., p. 182.

41. - TUILIER, André. Histoire de l'Université de Paris et de la Sorbonne, t. I. Paris : nouvelle librairie de France, G.-V. Labat éditeur, 1994, 620 p., p. 373.

42. - TUILIER, André. Histoire de l'Université de Paris et de la Sorbonne, t. I. Paris : nouvelle librairie de France, G.-V. Labat éditeur, 1994, 620 p., p. 387. 
43. - François $\mathrm{I}^{\mathrm{er}}$ avait envisagé, sans persévérer, de construire pour ses lecteurs royaux « un beau et grant collège ", sur le terrain de l'hôtel de Nesles, face au Louvre. Pierre Chambiges devait en être l'architecte. GADY, Alexandre. « Du Collège royal au Collège de France ». Dans Universités et grandes écoles à Paris. Les palais de la science. Paris: AAVP, 1999, 222 p., p. 79-88. Voir aussi : FUMAROLLI, Marc (dir.). Les origines du Collège de France (1500-1560). Paris, 1998 (Actes du colloque tenu à Paris en 1995).

44. - JOURDAIN, Ch. Histoire de l'Université de Paris au XVII et XVIII siècles. Paris, 1862, (réed. Bruxelles, 1966), p. 39-40.

45. - CAILLET, Maurice. "Le Collège des Irlandais ». Dans BACHA, Myriam et HOTTIN, Christian (dir.). Les bibliothèques parisiennes, architecture et décor. Paris, AAVP, 2002, 272 p., p. 81-82.

46. - TUILIER, André. Histoire de l'Université de Paris et de la Sorbonne, t. II. Paris : nouvelle librairie de France, G.-V. Labat éditeur, 1994, 657 p., p. 43-44.

47. - Bibliothèque de la Sorbonne, Ms. 243, fos $130-131^{\mathrm{v}}$.

48. - « Tous les humanistes et philosophes, qui jouissent actuellement ou qui jouiront dans la suite des bourses établies dans les différents collèges de cette ville de Paris, autres néanmoins que ceux d'Harcourt, de Cardinal Lemoyne, de Navarre, de Montaigu, du Plessis, de DormansBeauvais, de la Marche, des Grassins et de Mazarin, seront tenus, pour conserver les dites bourses, de fréquenter, à compter du $1^{\mathrm{er}}$ octobre prochain, les classes du Collège de Lisieux ». Bibliothèque de la Sorbonne, archives de l'Université de Paris, registre $50, f^{\circ} 20^{\mathrm{v}}$ et ROLLAND D'ERCEVILLE, B.G. Mémoire sur l'administration du Collège Louis-Le-Grand et des collèges y réunis. Paris, 1778, p. 158.

49. - Sur la situation des petits collèges parisiens au XVIII ${ }^{\mathrm{e}}$ siècle voir : TUILIER, André. Histoire de l'Université de Paris et de la Sorbonne, t. II. Paris : nouvelle librairie de France, G.-V. Labat éditeur, 1994, 657 p., p. 171-172. Ainsi, en 1763, les commissaires du Parlement chargés d'enquêter sur les collèges en vue de leur réforme recherchent en vain le principal du Collège de l'Ave Maria. Il était mort et nul ne songeait à le remplacer.

50. - TUILIER, André. Histoire de l'Université de Paris et de la Sorbonne, t. II. Paris : nouvelle librairie de France, G.-V. Labat éditeur, 1994, 657 p., p. 175.

51. - Voir le Mémoire pour la translation du Collège de Lisieux et le Supplément au mémoire pour la translation du Collège de Lisieux, cités par JOURDAIN, Ch. Histoire de l'Université de Paris au $\mathrm{XVII}^{\mathrm{e}}$ et XVIII ${ }^{\mathrm{e}}$ siècles. Paris, 1862, (réed. Bruxelles, 1966), p. 468, n. 57 et 58. La maison professe des jésuites est l'actuel lycée Charlemagne.

52. - JOURDAIN, Ch. Histoire de l'Université de Paris au XVII ${ }^{\mathrm{e}}$ et XVIII ${ }^{\mathrm{e}}$ siècles. Paris, 1862, (réed. Bruxelles, 1966), p. 468-469.

53. - Les plans sont montrés en 1772 à Louis XV, qui se borne à trouver l'édifice " fort beau ». JOURDAIN, Ch. Histoire de l'Université de Paris au XVII ${ }^{\mathrm{e}}$ et XVIII ${ }^{\mathrm{e}}$ siècles. Paris, 1862, (réed. Bruxelles, 1966), p. 450.

54. - Ce fut chose faite en 1775. Les médecins devaient rapidement déchanter devant la vétusté de leurs «nouvelles" installations. Ils demandèrent sans succès au roi leur déménagement à l'Hôpital Saint-Jacques. Le mémoire du doyen Charles Desessartz adressé au roi à ce sujet a été imprimé. TUILIER, André. Histoire de l'Université de Paris et de la Sorbonne, t. II. Paris : nouvelle librairie de France, G.-V. Labat éditeur, 1994, 657 p., p. 198.

55. - Par lettres patentes du 24 novembre 1769, enregistrées au Parlement le 2 décembre suivant. TUILIER, André, op. cit., p. 198-199.

56. - TUILIER, André, op. cit., p. 193-198.

57. - LAUGIER, Marc-Antoine. Essai et observations sur l'architecture. Bruxelles : Pierre Mardaga, 1979,316 et 326 p., p. 167-168.

58. - Sur Edme Verniquet, voir : PRONTEAU, Jeanne. Edme Verniquet (1727-1804), architecte et auteur du grand plan de Paris, Paris (1785-1791). Paris, 1986, 652 p. 
59. - La description qui suit est établie d'après l'exemple conservé à la BNF, département des imprimés, sous la cote GR FOL LK7 6043. Les planches étudiées sont les nº4, 45 et 53. VERNIQUET, Edme. Plan général de la ville de Paris. Paris, H.L. Perroneau, [1795], 4 p. de texte et $72 \mathrm{pl}$.

60. - VERNIQUET, Edme. Plan général de la ville de Paris. Paris : H.L. Perroneau, [1795], pl. 53, N 12.

61. - VERNIQUET, Edme, op. cit., pl. 45, M 14.

62. - Bien qu'indiqué sur le plan, le Collège de Picardie (VERNIQUET, Edme, op.cit., pl. 45, L 15) n'est plus actif à la fin de l'Ancien Régime.

63. - VERNIQUET, Edme, op. cit., pl. 53, N 12 et N 13.

64. - Reproduction du plan de la salle : TUILIER, André. Histoire de l'Université de Paris et de la Sorbonne, t. II. Paris : nouvelle librairie de France G.-V. Labat éditeur, 1994, 657 p., p. 149. Sur le bâtiment voir: De BEAUSCHENE. "Les écoles extérieures de la Sorbonne et la salle de distribution du concours général ». Dans Mémoires de la Société de l'Histoire de Paris et de l'île de France, Mélanges publiés à l'occasion du cinquantenaire de la Société, t. 49, 1927, p. 76-84.

65. - TUILIER, André, op. cit., p. 100.

66. - PONCELIN, Pierre. Histoire de Paris, et description de ses plus beaux monuments, Dessinés et gravés en taille douce par F.N. Martinet, ingénieur, dessinateur et graveur du cabinet du roi, t. III. Paris, 1781, XI-420, p. 171 (entrée et cour) et p. 182 (jardin). Les gravures de François Nicolas Martinet sont reproduites dans TUILIER, André, op. cit., p. 46 et 153.

67. - VERNIQUET, Edme, op. cit., pl. 53, N 12, O 12 et 013.

68. - PICON, Antoine. L'invention de l'ingénieur moderne; l'École des Ponts et Chaussées (1747-1851). Paris : Presses de l'École Nationale des Ponts et Chaussées, 1992, 767 p., p. 124-126.

69. - SAGE, Balthazar Georges. Origine de la création de l'École des Mines. Paris : Firmin Didot, 1813, 8 p., p. 4.

70. - JEANJOT-EMERY, Pol. «Historique ». Dans L'École Nationale Vétérinaire d'Alfort au XX $X^{e}$ siècle. Thionville : Gérard Klopp, 1998, 262 p. p. 19-27, p. 22.

71. - JEANJOT-EMERY, Pol, op. cit., p. 19-27, p. 22.

72. - Texte de la bulle Parens scientiarum, cité par VERGER, Jacques. Culture, enseignement et société en Occident au XIII siècle. Rennes : Presses universitaires de Rennes, date ?, 194 p., p. 125.

73. - JONGKEES, A.G. "Translatio studii : les avatars d'une théorie médiévale ». Dans Miscellanea mediaevalia in memoriam Jan Frederick Niermeyer. Groningue : J.B. Volters, 1967, p. 41-51.

74. - Voir: MERLIN, Pierre. L'Urbanisme universitaire à l'étranger et en France. Paris: Presses de l'École des Ponts et Chaussées, 1995, 416 p., plan reproduit p. 28.

75. - Marc Le Cœur a souligné la différence de richesse des établissements français et anglais en comparant les gravures de Martinet pour l'ouvrage de Pierre Poncelin et celles exécutées par Loggan pour l'Oxonia Illustrata : «On ne saurait mieux saisir la différence de considération dont jouissaient alors les installations des collèges de part et d'autre de la Manche qu'en confrontant ces modestes tailles-douces avec les majestueuses planches de Loggan, exécutées à Oxford et Cambridge, cent ans plus tôt ». LE CÆEUR, Marc. «Des collèges médiévaux aux campus ». Dans Histoire de l'Éducation, L'Architecture scolaire. Essai d'historiographie internationale, $\mathrm{n}^{\circ} 102$, mai 2004, p. 39-69, p. 51. 


\section{RÉSUMÉS}

Les cinquième et sixième arrondissements de Paris restent encore, en ce début de $\mathrm{XXI}^{\mathrm{e}}$ siècle, l'une des plus importantes concentrations d'établissements d'enseignement supérieur d'Europe. On a tenté d'expliquer, dans cet article, d'une part comment s'était constitué ce territoire, entre la fin du XII et la fin du XVIII ${ }^{\mathrm{e}}$ siècle, et d'autre part, on a souhaité montrer quelles étaient aujourd'hui encore les traces visibles des implantations de l'ancienne université de Paris : vestiges des collèges, des établissements religieux, des facultés, ou des institutions extérieures à l'Université.

\section{INDEX}

Mots-clés : architecture, urbanisme, université, collège, enseignement supérieur, Paris, Moyen Âge, Ancien Régime, géographie historique

\section{AUTEUR}

\section{CHRISTIAN HOTTIN}

Conservateur du patrimoine, adjoint, département du pilotage de la recherche et de la politique scientifique, direction générale des patrimoines, ministère de la Culture et de la Communication christian.hottin@culture.gouv.fr 